\title{
Learning and Adaptation in a Recurrent Model of V1 Orientation Selectivity
}

\author{
Andrew F. Teich and Ning Qian \\ Center for Neurobiology and Behavior and Department of Physiology and Cellular Biophysics, Columbia University, New York, New York 10032
}

Submitted 28 October 2002; accepted in final form 10 December 2002

Teich, Andrew F. and Ning Qian. Learning and adaptation in a recurrent model of V1 orientation selectivity. J Neurophysiol 89: 2086-2100, 2003. First published December 18, 2002; 10.1152/jn.00970.2002. Learning and adaptation in the domain of orientation processing are among the most studied topics in the literature. However, little effort has been devoted to explaining the diverse array of experimental findings via a physiologically based model. We have started to address this issue in the framework of the recurrent model of V1 orientation selectivity and found that reported changes in V1 orientation tuning curves after learning and adaptation can both be explained with the model. Specifically, the sharpening of orientation tuning curves near the trained orientation after learning can be accounted for by slightly reducing net excitatory connections to cells around the trained orientation, while the broadening and peak shift of the tuning curves after adaptation can be reproduced by appropriately scaling down both excitation and inhibition around the adapted orientation. In addition, we investigated the perceptual consequences of the tuning curve changes induced by learning and adaptation using signal detection theory. We found that in the case of learning, the physiological changes can account for the psychophysical data well. In the case of adaptation, however, there is a clear discrepancy between the psychophysical data from alert human subjects and the physiological data from anesthetized animals. Instead, human adaptation studies can be better accounted for by the learning data from behaving animals. Our work suggests that adaptation in behaving subjects may be viewed as a short-term form of learning.

\section{N T R O D U C T I O N}

It is well known that orientation discrimination, like most other visual discrimination tasks, is subject to learning: One can reliably detect significantly smaller orientational differences after being trained on the task over an extended period of time (Gilbert 1994; Schoups et al. 1995; Shiu and Pashler 1992; Vogels and Orban 1985). The performance improvement of this perceptual learning phenomenon is long-lasting, indicating that the training process must lead to some form of long-term changes in the brain. The psychophysically observed specificities of perceptual learning, such as the lack of transfer from learning at one orientation to the orthogonal orientation or from one learned retinal location to a nearby nonoverlapping location, strongly suggest that the site of plasticity must involve, at least partially, early visual cortical areas where cells have relatively narrow orientation tuning curves and small receptive fields (Gilbert 1994). When two independent groups recorded from monkey V1 cells after training monkeys on orientation discrimination tasks, they indeed found a longlasting change in V1. The result, however, was puzzling ini-

Address for reprint requests: N. Qian, Center for Neurobiology and Behavior, Columbia University, P.I. Annex Rm. 730, 722 W. 168th St., New York, NY 10032 (E-mail: nq6@ columbia.edu). tially: both groups found that the main effect of learning was a firing-rate reduction for the cells tuned at and around the trained orientation (Ghose and Maunsell 1997; Schoups et al. 1998); changes that could obviously explain learning, such as a reduced variability of neuronal firing, were not observed. In an effort to understand this finding, we reported previously in abstract form (Qian and Matthews 1999) that when the observed activity reduction is introduced into the recurrent model of V1 orientation selectivity (Ben-Yishai et al. 1995; Carandini and Ringach 1997; Douglas et al. 1995; Somers et al. 1995) through changes of connection strengths, cells with preferred orientations close to the trained orientation should have sharper tuning at the trained orientation, while cells with preferred orientations somewhat further away should have broader tuning curves. Furthermore, these tuning-curve changes are precisely what is needed for improving orientation discrimination at the trained orientation. On re-analyzing the data in light of the model, Schoups et al. (2001) indeed found the predicted changes in orientation tuning. In this paper, we present a full account of our model with extensive simulations and provide a detailed comparison with the relevant experimental data, particularly from Schoups et al. (2001).

The observed activity reduction after learning also suggests that learning may be related to adaptation, since it is well known that neurons tuned at and around the adapted orientation have reduced firing rates. One obvious difference is that the activity reduction after adaptation is transient, whereas the reduction seems to be permanent after learning (Ghose and Maunsell 1997; Schoups et al. 1998). However, this may simply mean that learning is a more permanent version of adaptation. The possible link between learning and adaptation is strengthened by an earlier psychophysical finding that adaptation, like learning, can also improve orientation discrimination at the adapted orientation, albeit only briefly (Regan and Beverley 1985). However, a recent physiological experiment on anesthetized cats by Dragoi et al. (2000) showed that, unlike learning, the V1 orientation tuning curves after adaptation became broader for cells tuned around the adapted orientation. In addition, the peak of these cells' tuning curves shifted away from the adapted orientation. Here we show that these physiological changes to the orientation tuning after adaptation can also be explained with the recurrent model by altering the connection strengths in a different way. Thus, just as we will compare our learning simulations to the learning study of Schoups et al. (2001), we will also compare our adaptation simulations to the adaptation study of Dragoi et al. (2000).

\footnotetext{
The costs of publication of this article were defrayed in part by the payment of page charges. The article must therefore be hereby marked "advertisement" in accordance with 18 U.S.C. Section 1734 solely to indicate this fact.
} 
While the behavioral consequence of learning is consistent with the physiologically observed tuning curve changes, in the case of adaptation we found that the physiological data from anesthetized animals cannot explain the psychophysical observations on alert, attending human subjects. We will discuss the relevant computational, psychophysical, and physiological results in this paper. A conclusion emerging from this discussion is that orientation adaptation in alert human subjects may be better viewed as a short-term form of orientation learning.

\section{METHODS}

All simulations were performed with Matlab (Mathworks, Natick, MA) on a Linux computer. The source code, which is modified from that of Carandini and Ringach (1997), will be available to anyone interested.

\section{Simulating learning and adaptation in a recurrent model}

We did not model the time course of synaptic modifications during the process of learning or adaptation. Instead, we focused on how the end effects of learning and adaptation on orientation tuning curves that are found physiologically may be understood by changing the connection strengths in the recurrent model of V1 orientation selectivity (Ben-Yishai et al. 1995; Carandini and Ringach 1997; Douglas et al. 1995; Somers et al. 1995). Among the published versions of the recurrent model, that of Somers et al. (1995) is the most anatomically and physiologically accurate. Their model was subsequently simplified by Carandini and Ringach (1997) to the most essential ingredients. For computational efficiency, we based our work on the simplified model of Carandini and Ringach (1997). Briefly, the model considers $N$ oriented V1 cells with their preferred orientations $(\theta)$, evenly distributed in the entire $180^{\circ}$ range. These cells receive feedforward inputs that are weakly orientation-biased, as illustrated in Fig. $1 A$ (for cats, the feed-forward inputs are from spatially aligned LGN activities, while for monkeys, they may also include contributions from center-surround cells in layer $4 \mathrm{C}$ of V1). To achieve strong orientation tuning typically observed in $\mathrm{V} 1$, recurrent excitatory and inhibitory connections are introduced among the model V1 cells. Following the experimental evidence (Ferster 1986; Michalski et al. 1983), both excitatory and inhibitory connections in the model are strongest among cells with the same preferred orientation, and they drop off with increasing difference between the preferred orientations of the cells (Fig. 1B). The model requires that the inhibitory connectivity be wider than the excitatory connectivity, such that the net interaction among the V1 cells follows a "Mexican hat" type of profile (Fig. 1C). This interaction profile can sharpen the weak orientation bias in the feed-forward input into the typical V1 orientation tuning curves (Fig. 1D).

Quantitatively (Carandini and Ringach 1997), the membrane potential $V(\theta, \phi, t)$ of a cell with preferred orientation $\theta$, responding to a stimulus orientation $\phi$, obeys the equation

$$
\tau \partial V / \partial t+V=V_{\mathrm{f}}+V_{\mathrm{e}}-V_{\mathrm{i}}
$$

where $\tau$ is the membrane time constant, and $V_{\mathrm{f}}, V_{\mathrm{e}}$, and $V_{\mathrm{i}}$ are the synaptic potentials generated by the feed-forward, recurrent excitatory, and recurrent inhibitory inputs to the cell, respectively. The cell's firing rate is assumed to be related to its membrane potential via the threshold function with a gain factor $\alpha$

$$
R=\alpha \max (V, 0)
$$

Note that for simplicity, $V$ is measured relative to the firing threshold and $R$ is measured relative to the spontaneous firing rate. When a stimulus orientation $\phi$ is presented, the feed-forward input to the cell with preferred orientation $\theta$ takes the form

$$
V_{\mathrm{f}}(\theta, \phi)=J_{\mathrm{f}}{ }^{\circ} \exp \left[-(\theta-\phi)^{2} /\left(2 \sigma_{\mathrm{f}}^{2}\right)\right]
$$

where $J_{\mathrm{f}}^{\circ}$ and $\sigma_{\mathrm{f}}$ determine the strength and width of the input. The synaptic potentials from recurrent connections to a given cell with preferred orientation $\theta$ are determined by integrating contributions
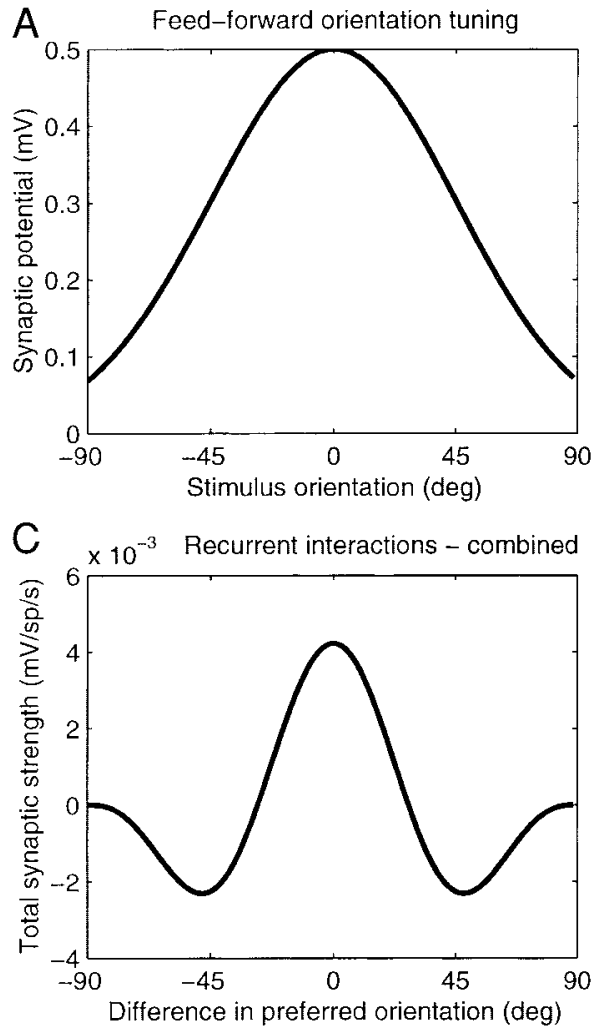
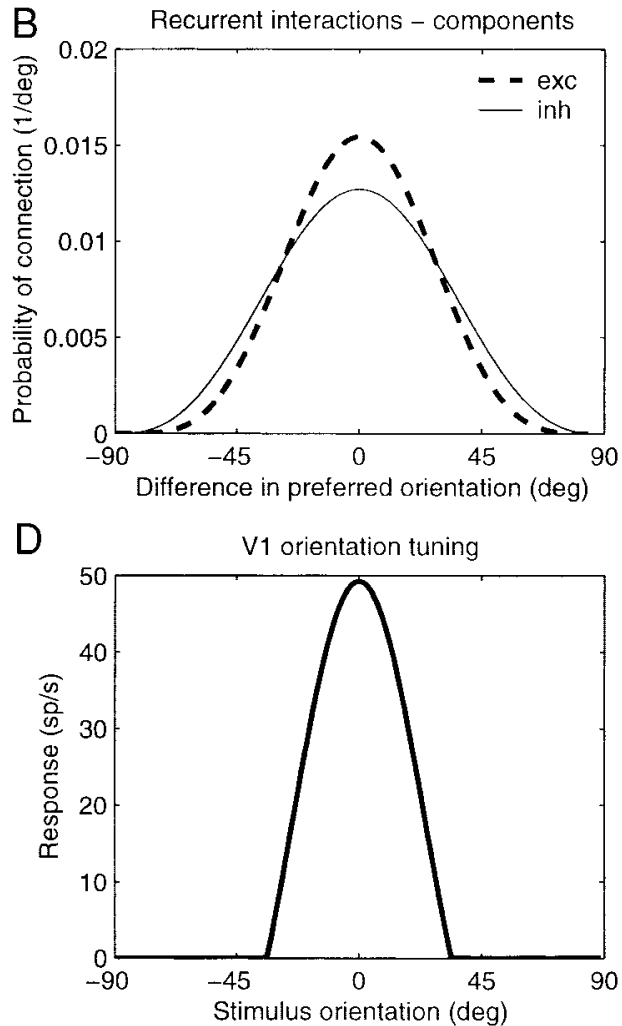

FIG. 1. Baseline simulation of V1 orientation tuning in a recurrent model. $A$ : feedforward, weakly orientation-biased inputs to the V1 cells. $B$ : distribution of recurrent excitatory (exc) and recurrent inhibitory (inh) connections between 2 cells as a function of the difference between the cells' preferred orientations. $C$ : total recurrent interaction profile, generated as $\left[J_{\mathrm{e}}^{\circ} E(\theta)-J_{\mathrm{i}}{ }^{\circ} I(\theta)\right] . D$ : orientation tuning curve for a V1 cell, with $0^{\circ}$ preferred orientation. 
TABLE 1. Parameters used in the simulations

\begin{tabular}{ll}
\hline \hline$N$ & Number of cells in the network (128) \\
$\tau$ & Membrane time constant (15 ms) \\
$\Delta t$ & Time step of integration $(2 \mathrm{~ms})$ \\
$\alpha$ & Gain of spike encoder (10 spikes/s/mV) \\
$J_{\mathrm{e}}^{\circ}$ & Strength of intracortical excitation $(1.1 \mathrm{mV} / \mathrm{spikes} / \mathrm{s})$ \\
$J_{\mathrm{i}}^{\circ}$ & Strength of intracortical inhibition $(1.1 \mathrm{mV} / \mathrm{spikes} / \mathrm{s})$ \\
$J_{\mathrm{f}}^{\circ}$ & Strength of feed-forward excitation $(1.5 \mathrm{mV} / \mathrm{spikes} / \mathrm{s})$ \\
$\sigma_{\mathrm{f}}$ & Gaussian width of feed-forward orientation bias $\left(45^{\circ}\right)$ \\
$a_{\mathrm{e}}$ & Exponent for excitatory connection distribution $(2.2)$ \\
$a_{\mathrm{i}}$ & Exponent for inhibitory connection distribution $(1.4)$ \\
$A_{\mathrm{e}}$ & Fractional reduction of $J_{\mathrm{e}}^{\circ}$ at the trained or adapted orientation \\
& (ranged from 0.005 to 0.015 in our learning simulations and \\
& from 0.1 to 0.4 in our adaptation simulations) \\
$A_{\mathrm{i}}$ & Fractional reduction of $J_{\mathrm{i}}^{\circ}$ at the trained or adapted orientation \\
& (was zero in our learning simulations, and was 0.01 to 0.03 \\
& larger than the corresponding $A_{\mathrm{e}}$ in our adaptation simulations) \\
$\sigma_{\mathrm{r}}$ & Spread of the change in input connections at the trained or adapted \\
& orientation to other orientations (ranged from $20^{\circ}$ to $\left.26^{\circ}\right)$ \\
\hline
\end{tabular}

from all cells with preferred orientations $\theta^{\prime}$ covering the entire $180^{\circ}$ range

$$
\begin{aligned}
& V_{\mathrm{e}}(\theta, t)=J_{\mathrm{e}}^{\circ} \int_{-90^{\circ}}^{90^{\circ}} E\left(\theta-\theta^{\prime}\right) R\left(\theta^{\prime}, t\right) d \theta^{\prime} \\
& V_{\mathrm{i}}(\theta, t)=J_{\mathrm{i}}^{\circ} \int_{-90^{\circ}}^{90^{\circ}} I\left(\theta-\theta^{\prime}\right) R\left(\theta^{\prime}, t\right) d \theta^{\prime}
\end{aligned}
$$

where $J_{\mathrm{e}}$ and $J_{\mathrm{i}}$ determine the strengths of excitatory and inhibitory interactions, and $E(\theta)$ and $I(\theta)$ are the corresponding connection probabilities. For a given broadly tuned feed-forward input $V_{\mathrm{f}}$, the firing rate $R$ will evolve through time according to Eqs. 1 and 2 into sharp orientation tuning found in V1 (Carandini and Ringach 1997).

We made a few modifications to the model of Carandini and Ringach (1997) without changing its essential features. First, we did not truncate the recurrent connection probabilities $E(\theta)$ and $I(\theta)$ at $\pm 60^{\circ}$, because biological systems are unlikely to enforce such an abrupt cutoff. Instead, we used the following periodic functions for the connection probabilities

$$
\begin{gathered}
E(\theta)=c_{\mathrm{e}}[\cos (2 \theta)+1]^{\mathrm{a}_{\mathrm{e}}} \\
I(\theta)=c_{\mathrm{i}}[\cos (2 \theta)+1]^{\mathrm{a}_{\mathrm{i}}}
\end{gathered}
$$

where $c_{\mathrm{e}}$ and $c_{\mathrm{i}}$ are the normalization factors for making the total $E$ and $I$ to a given cell unity, and the exponents $a_{\mathrm{e}}$ and $a_{\mathrm{i}}$ determine the sharpness of the distributions. Second, in the original model, the inhibitory profile is much wider than the excitatory profile. This has been the source of some criticism because physiological data suggest that the difference between the two profiles may be small (Ferster 1986). We therefore narrowed the difference (Fig. 1B) in our simulations. Finally, the V1 orientation tuning widths in the simulations of Carandini and Ringach (1997) are too sharp compared with typical V1 cells. We adjusted the parameters in the model so that the full width at the half-height is about $40^{\circ}$ (Fig. $1 D$ ). The standard set of parameters used in our simulations is listed in Table 1. Figure 1 shows that the modified model works well in generating V1 orientation tuning curves by sharpening the feed-forward inputs.

Our modification of Carandini and Ringach (1997) also eliminates a major problem with their model - the appearance of spurious peaks at nonoptimal orientations when noise is introduced into the feedforward input. The problem is serious because noise is an inevitable component in any neural system. We have made extensive simulations and have never seen spurious peaks with our parameter set. The reason is that the parameters used by Carandini and Ringach (1997) generate tuning curves with a $20^{\circ}$ full width at half-height, twice as sharp as typical V1 tuning curves. To obtain such sharp curves, they had to use a sharp excitatory interaction profile, which makes the system less stable. In contrast, we used a more diffuse excitatory profile (Ferster 1986) to generate tuning curves with a $40^{\circ}$ full width at half-height, and the system is more stable. In Fig. 2, we show our simulations with two different noise levels. No spurious peaks appear

\section{Ten Percent Noise}
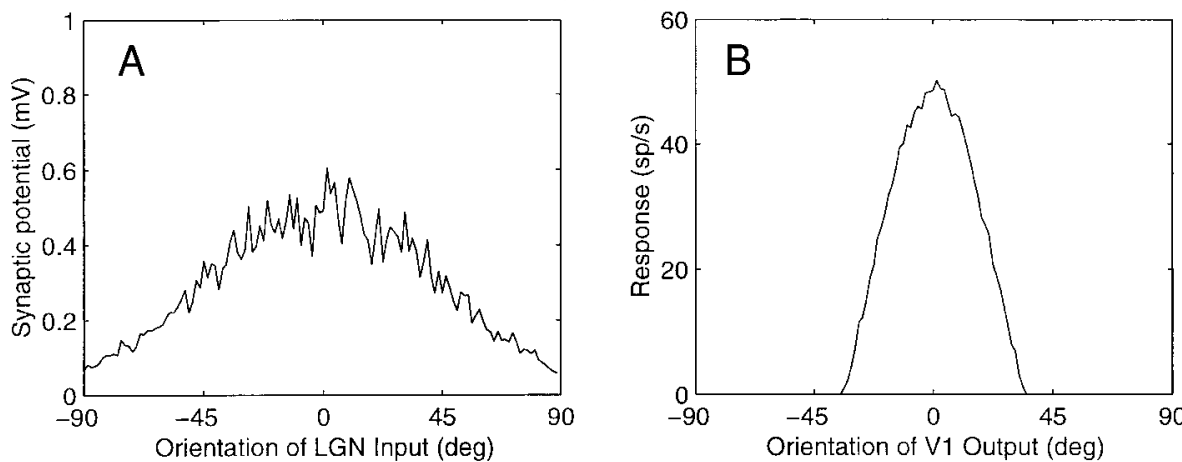

Twenty-Five Percent Noise
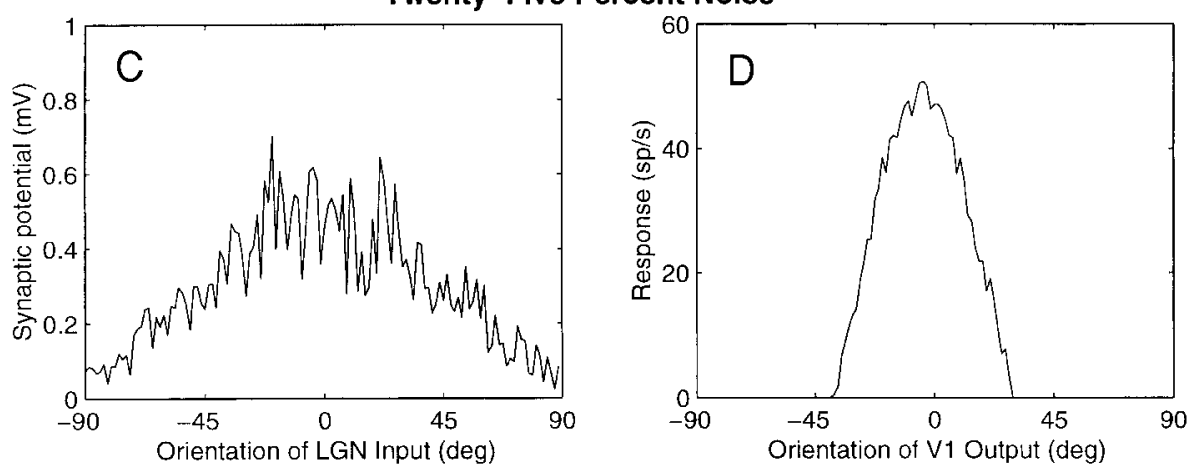

J Neurophysiol $\bullet$ VOL $89 \cdot$ APRIL $2003 \cdot$ www.jn.org
FIG. 2. Noise in the recurrent model. $A$ : feedforward input with $10 \%$ noise. $B$ : corresponding V1 tuning curve. $C$ : feed-forward input with $25 \%$ noise. $D$ : corresponding V1 tuning curve. In each case, noise was introduced by selecting each feedforward input value from a Gaussian distribution, with the mean set to the original, noise-free value and the SD set to 0.1 or 0.25 times the mean. In neither case did spurious peaks appear at nonoptimal orientations. 
even with noise much greater than those introduced by Carandini and Ringach (1997).

Figure 1 shows the baseline behavior of the model before learning or adaptation. We now describe how we modeled learning and adaptation by modifying connections in this framework. Obviously, we have to assume that the adaptation induced changes are short term while the learning induced changes are long-lasting. Without loss of generality, we denote the learned or adapted orientation $0^{\circ}$. We found that the easiest way to simulate the learning data of Schoups et al. (2001) was to either reduce the excitatory recurrent connections or increase the recurrent inhibitory connections to cells around the trained orientation by a small amount. For the excitation manipulation, we replaced the constant $J_{\mathrm{e}}{ }^{\circ}$ in $E q .4$ by a function of the preferred orientation $\theta$ of the postsynaptic cell

$$
J_{\mathrm{e}}(\theta)=J_{\mathrm{e}}{ }^{\circ}\left(1-A_{\mathrm{e}} \exp \left[-\theta^{2} /\left(2 \sigma_{\mathrm{r}}^{2}\right)\right]\right)
$$

where $A_{\mathrm{e}}$, a small positive fractional number, is the fractional reduction of the connection strength at the trained orientation $\left(\theta=0^{\circ}\right)$, and $\sigma_{\mathrm{r}}$ determines the spread of reduction around the trained orientation. Likewise, for the inhibition manipulation, we replaced the constant $J_{i}{ }^{\circ}$ in Eq. 5 by

$$
J_{\mathrm{i}}(\theta)=J_{\mathrm{i}}^{\circ}\left(1-A_{\mathrm{i}} \exp \left[-\theta^{2} /\left(2 \sigma_{\mathrm{r}}^{2}\right)\right]\right)
$$

Since inhibition should be slightly increased, $A_{\mathrm{i}}$ should be a small negative fractional number in this option. As a third alternative, we could also simulate the learning data by reducing both recurrent excitatory and inhibitory connections (i.e., letting both $A_{\mathrm{e}}$ and $A_{\mathrm{i}}$ be positive fractional numbers), as long as $A_{\mathrm{e}}$ was slightly larger than $A_{\mathrm{i}}$. Since these three manipulations generated essentially the same changes to the tuning curves, we will only report results from a simple reduction in excitatory recurrent connections. The range of $A_{\mathrm{e}}$ used in our learning simulations is listed in Table 1 , whereas $A_{\mathrm{i}}$ was kept at zero.

We were able to simulate the adaptation data of Dragoi et al. (2000) by decreasing both recurrent excitatory and recurrent inhibitory connections to cells around the adapted orientation by a large fraction, i.e., by setting both $A_{\mathrm{e}}$ and $A_{\mathrm{i}}$ to large, positive fractional numbers. In addition, unlike the third alternative for the learning simulation mentioned above, $A_{\mathrm{i}}$ had to be slightly larger than $A_{\mathrm{e}}$ to simulate the adaptation data. The ranges of $A_{\mathrm{e}}$ and $A_{\mathrm{i}}$ used in our adaptation simulations are listed in Table 1 . Note that $A_{\mathrm{e}}$ and $A_{\mathrm{i}}$ only determine the percentage reductions of the connection strengths from the original values, but are not the actual amounts of reduction. Since the inhibitory interaction profile had a lower peak and higher flanks than that of excitation (Fig. $1 B$ ), the effect of introducing $A_{\mathrm{e}}$ and $A_{\mathrm{i}}$ in our adaptation simulations was actually a slight decrease of net excitation (i.e., reduced peak amplitude of the Mexican-hat interaction profile) to cells around the adapted orientation just like the learning case; in addition, there was a small decrease of net side inhibition (i.e., reduced trough amplitudes of the Mexican-hat interaction profiles). We will provide intuitive explanations of why our learning and adaptation manipulations can explain the observed physiological data of Schoups et al. (2001) and Dragoi et al. (2000), respectively, in RESULTS. Note that although the net connection changes for the learning and adaptation simulations differ, in both cases the largest changes to the excitatory $\left(J_{\mathrm{e}}\right)$ and inhibitory $\left(J_{\mathrm{i}}\right)$ connections occur for cells tuned to the trained or adapted orientation.

For each cell with preferred orientation $\theta$ and stimulus orientation $\phi$, we can apply the above equations to obtain a steady-state response $R(\theta, \phi)$. If we plot $R(\psi, \phi)$ as a function of $\phi$ for a fixed $\theta=\psi$, we obtain the orientation tuning curve of the model V1 cell with the preferred orientation $\psi$. On the other hand, if we plot $R(\theta, \psi)$ as a function of $\theta$ for a fixed $\phi=\psi$, we get the population response of all model V1 cells in the network to a given stimulus orientation $\psi$. Note that before introducing learning- or adaptation-induced changes in the model, the tuning curve $R(\psi, \phi)$ and the population response $R(\theta, \psi)$ have exactly the same shape. This is because the symmetry of the network ensures that

$$
R(\theta, \phi)=R(\theta-\phi)=R(\phi-\theta)=R(|\phi-\theta|)
$$

Therefore $R(\psi, \phi)$ as a function of $\phi$ is the same as $R(\theta, \psi)$ as a function of $\theta$. For this reason, to obtain the orientation tuning curve of the cell preferring the $0^{\circ}$ orientation shown in Fig. $1 D$, we can simply compute the population response of all cells to a $0^{\circ}$ stimulus orientation. However, the symmetry of the network is broken when Eqs. 8 and 9 are used for modeling learning and adaptation. In these cases, the population responses and orientation tuning curves no longer have identical shapes. This point will be important when we discuss the psychophysical implications of learning and adaptation.

Since the orientation tuning in the recurrent model emerges with time, we need to determine how many iterations to run the network in our numerical simulations before plotting the results. For all simulations with the standard parameter set, we report results from 500 iterations (corresponding to $1 \mathrm{~s}$, since we used a step of $2 \mathrm{~ms}$ for integrating $E q$. l) for two reasons. First, the network typically evolves very quickly in the first 300 iterations, and by 500 iterations, the bulk of the changes have already occurred. For the baseline simulation (without learning or adaptation), the difference between the tuning width at 500 and 2,000 iterations is $<0.001 \%$, and the difference between the peak firing rates is $<0.002 \%$. For the leaning simulations, the difference between 500 and 2,000 iterations for the average tuning width and peak firing rate is $<0.6 \%$ and $0.3 \%$, respectively. For the adaptation simulations, the difference is $<0.4 \%$ for the average tuning width and $<0.08 \%$ for the peak firing rates. Second, we are only interested in the time scale comparable to that of the relevant psychophysical and physiological experiments. Although the process of learning and adaptation can take a long time, the testing phase after learning or adaptation for measuring subjects' orientation discriminability or cells' firing rates only involve a brief stimulus presentation (typically from 200 to $1,000 \mathrm{~ms}$ ) in each trial.

For the learning simulations, our standard parameter set contains $A_{\mathrm{e}}$ values ranging from 0.005 to 0.015 and $\sigma_{\mathrm{r}}$ values ranging from 20 to 26 . For the adaptation simulations, our standard parameter set contains the same $\sigma_{\mathrm{r}}$ range as the learning simulations (20-26), and $A_{\mathrm{e}}$ values ranging from 0.1 to 0.4 . In the adaptation simulations, each $A_{\mathrm{e}}$ and $\sigma_{\mathrm{r}}$ combination has a corresponding $A_{\mathrm{i}}$ value that is approximately 5-10\% more than the $A_{\mathrm{e}}$ value; as both $A_{\mathrm{e}}$ and $\sigma_{\mathrm{r}}$ increase, the required $A_{\mathrm{i}}$ value also increases. For a given $A_{\mathrm{e}}, A_{\mathrm{i}}, \sigma_{\mathrm{r}}$ combination, $A_{\mathrm{i}}$ can generally be increased an additional $5-10 \%$ above the standard range, and the simulation will continue to match the data of Dragoi et al. (2000)

In addition to this standard set, we also made extensive simulations to explore the effects of lowering $\sigma_{\mathrm{r}}$ values (to as low as 4) in both the learning and the adaptation simulation. Lower $\sigma_{\mathrm{r}}$ values generally yielded the same behavior from the model at 500 iterations except that the range of activity reduction and the consequent tuning curve changes around the trained or adapted orientation were reduced, as expected. However, if the simulations for learning were run to 2,000 iterations, certain $A_{\mathrm{e}}$ values paired with low $\sigma_{\mathrm{r}}$ values yielded orientation tuning curves with an abnormally pointed apex. Also, in the adaptation simulations, low $\sigma_{\mathrm{r}}$ values yielded orientation tuning curves that curve and bend in slightly unusual shapes. These abnormalities do not make any qualitative differences. However, we will focus on the standard parameter set in this paper as these parameter combinations yielded simulations with no complications.

\section{Signal detection theory for orientation discrimination}

We used signal detection theory (Green and Swets 1966) to relate cells' population responses to a performance measure for an orientation discrimination task. For convenience of description, we use subscript i to label cells with different preferred orientations, and $r_{\mathrm{i}}(\phi)$ 
to denote the $i$ th cell's mean firing rate (spikes/s) to stimulus orientation $\phi$. The mean firing rate can be read from the cell's orientation tuning curve.

The mean number of spikes of cell $i$ to stimulus orientation $\phi$ over the duration $\Delta t$ is therefore $m_{\mathrm{i}}(\phi)=r_{\mathrm{i}}(\phi) \Delta t$. The actual activity in a given trial, however, is highly variable. It has been shown that the variance of the number of spikes under identical stimulus conditions is proportional to the mean spike count: $\operatorname{var}_{i}(\phi)=k m_{\mathrm{i}}(\phi)$, where $k$ is a dimensionless constant found to be between 1 and 4 (Peres and Hochstein 1994; Shadlen and Newsome 1994; Snowden et al. 1992; Softky and Koch 1993), and we used a value of 2 in our simulations. We can therefore simulate the actual activity of cell $i$ in a trial according to

$$
x_{\mathrm{i}}(\phi)=\mathcal{N}\left[m_{\mathrm{i}}(\phi), \sqrt{k m_{\mathrm{i}}(\phi)}\right]
$$

where $\mathcal{N}(\mu, \sigma)$ is a random variable following a normal distribution of mean $\mu$ and SD $\sigma$ (we did not use a Poisson distribution because it would force $k=1$ ).

To simulate psychophysical experiments of orientation discrimination, we will assume that two slightly different orientations, $\phi_{1}$ and $\phi_{2}$, are presented sequentially in a trial, each for a duration of $\Delta t$. Two sets of population responses will be generated among the cells: $\mathbf{x}\left(\phi_{\mathrm{j}}\right)=\left[x_{\mathrm{i}}\left(\phi_{\mathrm{j}}\right)\right] ; j=1,2$. In signal detection theory, each cell determines whether the two successive orientations in a trial form a clockwise or counterclockwise rotation by comparing its responses to the two orientations (Green and Swets 1966; Lehky and Sejnowski 1990). For cell $i$ whose mean response to $\phi_{1}$ is larger (smaller) than to $\phi_{2}$, it will make a correct decision when $x_{\mathrm{i}}\left(\phi_{1}\right)$ is larger (smaller) than $x_{\mathrm{i}}\left(\phi_{2}\right)$ and an incorrect decision otherwise. (Strictly speaking, this is only true when $\phi_{1}$ and $\phi_{2}$ always fall on the same side of the cell's tuning curve so that the tuning function can be considered monotonic and the cell "knows" the right response based on its mean activities to the 2 orientations. Since the difference between $\phi_{1}$ and $\phi_{2}$ $\left(1^{\circ}-2^{\circ}\right)$ is much smaller than a typical orientation tuning width (about $40^{\circ}$ ), this is a reasonable assumption.) A pooling across all cells in the population will then be performed to reach a final decision.

Since the response $x_{\mathrm{i}}\left(\phi_{\mathrm{j}}\right)$ of cell $i$ to stimulus orientation $\phi_{\mathrm{j}}$ follows a normal distribution $N\left[m_{\mathrm{i}}\left(\phi_{\mathrm{j}}\right), \sqrt{k m_{1}\left(\phi_{\mathrm{j}}\right)}\right]$, the difference $\left[x_{\mathrm{i}}\left(\phi_{1}\right)-\right.$ $x_{\mathrm{i}}\left(\phi_{2}\right)$ ] also follows a normal distribution with mean $\left[m_{\mathrm{i}}\left(\phi_{1}\right)-\right.$ $\left.m_{\mathrm{i}}\left(\phi_{2}\right)\right]$ and SD $\sqrt{k\left[m_{\mathrm{i}}\left(\phi_{1}\right)+m_{\mathrm{i}}\left(\phi_{2}\right)\right]}$. By combining the cases of $\left[m_{\mathrm{i}}\left(\phi_{1}\right)-m_{\mathrm{i}}\left(\phi_{2}\right)\right]>0$ and $<0$, we can express the probability of a correct decision as (Green and Swets 1966; Lehky and Sejnowski 1990)

$$
p_{\mathrm{i}}=\frac{1}{2} \operatorname{erfc}\left(\frac{-d^{\prime}}{\sqrt{2}}\right) ; \quad \text { with } \quad d^{\prime}=\frac{\left|m_{\mathrm{i}}\left(\phi_{1}\right)-m_{\mathrm{i}}\left(\phi_{2}\right)\right|}{\sqrt{k\left[m_{\mathrm{i}}\left(\phi_{1}\right)+m_{\mathrm{i}}\left(\phi_{2}\right)\right]}}
$$

where $\operatorname{erfc}(\cdot)$ is the standard complementary error function whose definition ensures that the value of $p_{\mathrm{i}}$ is between 0.5 (chance) and 1 (perfect). The final decision of the system is based on a majority vote of all cells: if more than one-half of the cells make a correct decision, the final decision is assumed to be correct in that trial; otherwise it is incorrect. By repeating this calculation over many trials, we can obtain a percent correct measure for the orientation discrimination task.

\section{RES U L T S}

\section{Learning}

As mentioned above, we simulated orientation tuning of V1 cells before and after learning using the simplified recurrent model of Carandini and Ringach (1997). The model assumes that V1 cells receive a broadly tuned feed-forward input that is subsequently sharpened by intracortical excitation and inhibition (Fig. 1). Before learning, the orientation tuning curves of the model cells were all identical in shape but shifted with respect to each other in peak location (Fig. 3A). Each curve can be viewed as representing the average tuning curve of all cells with the same preferred orientation for a given spatial location. Since it was first reported that the main consequence of orientation learning was a reduction of neuronal activities around the trained orientation (Ghose and Maunsell 1997; Schoups et al. 1998), we simulated this end effect of learning by slightly depressing intracortical excitatory connections to cells at and near the trained orientation (see METHODS). As expected, this manipulation reduced the responses of the model cells around the trained orientation (Fig. 3B), consistent with the physiological reports. To generate this figure, the excitatory connections only needed to be reduced by $0.75 \%$ at the trained orientation; this led to a $20 \%$ reduction of the neuronal activity at the trained orientation. For the parameter ranges in our standard parameter set (Table 1), the maximum activity reduction (occurring at the trained orientation) varied from $13 \%$ to $35 \%$. Physiologically reported maximum reduction values range from $10 \%$ to $30 \%$ (Ghose and Maunsell 1997; Schoups et al. 1998).

In addition to response depression, there are also changes to the shape of orientation tuning curves. Specifically, curve narrowing and peak shifting were observed for model cells whose preferred orientations were near the learned orientation. In Fig. $4 A$, the orientation tuning curves of such a cell before learning (dashed) and after learning (solid) are shown. The peak shifting was toward the learned orientation; this made the postlearning tuning curves asymmetrical, with sharpening on the side facing the learned orientation. In contrast, orientation tuning curves of cells with preferred orientations somewhat further away from the learned orientation broadened modestly

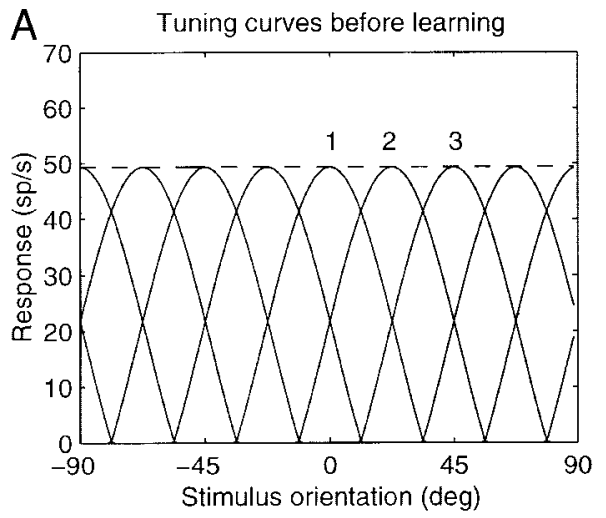

FIG. 3. Simulation of learning in a recurrent model. A: orientation tuning curves for a subset of model V1 cells before learning. Dashed line was derived by plotting the peak response of every cell in the network. $B$ : orientation tuning curves for the same subset of model cells after simulating learning at $0^{\circ}$. Peak response curve (dashed) shows a dip at the learned orientation. This figure was obtained with an $A_{\mathrm{e}}$ of 0.0075 and a $\sigma_{\mathrm{r}}$ of 24 . 
A
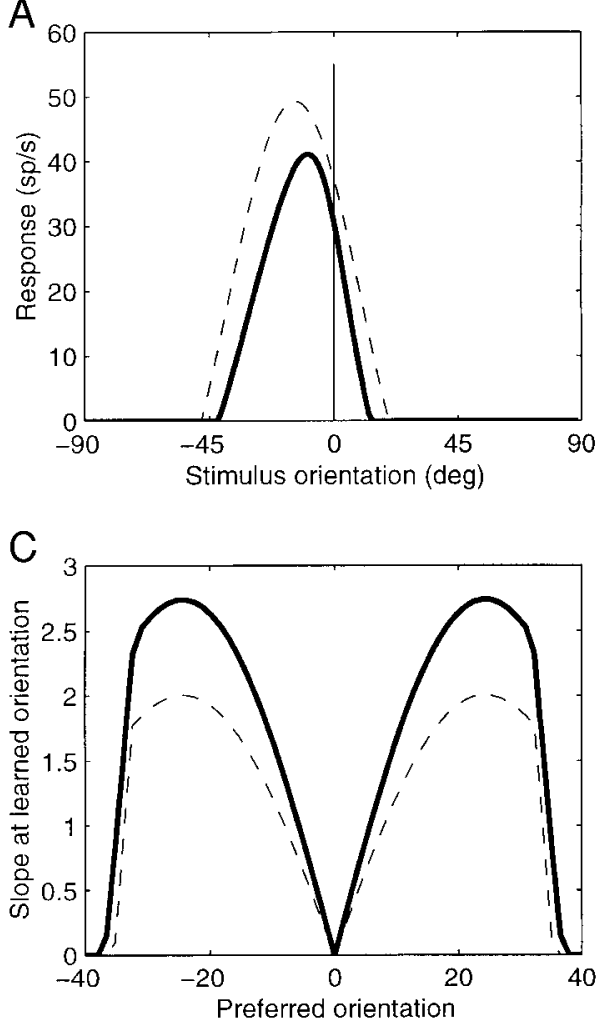

B

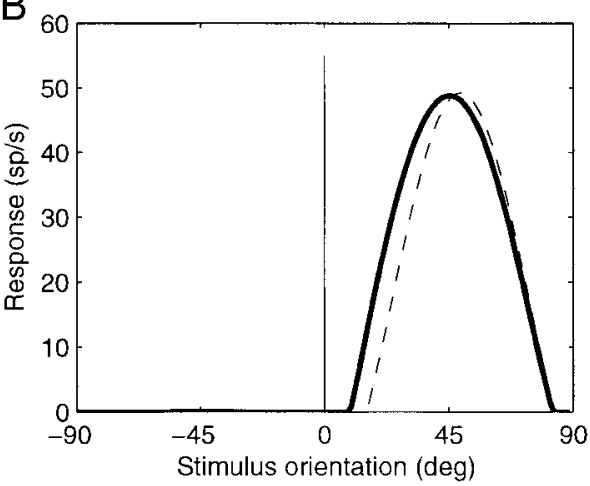

$\mathrm{D}$

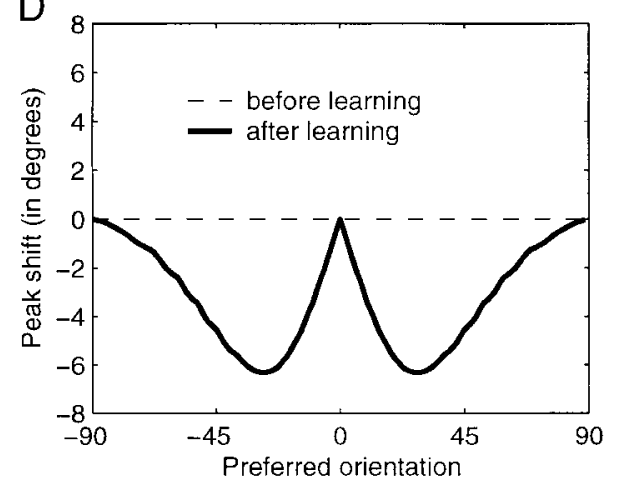

FIG. 4. Effects of learning in the recurrent model. This figure makes the effects of the learning simulation in Fig. 3 more explicit. Learning has been simulated at $0^{\circ}$ (marked by vertical lines). In all panels, dashed and solid lines represent the prelearning and postlearning conditions, respectively. $A$ : pre- and postlearning orientation tuning curves of a cell whose preferred orientation $\left(-14^{\circ}\right)$ is close to the learned orientation. Learning causes sharpening of the tuning curve, with a higher slope on the side facing the learned orientation, and a shift of peak toward the learned orientation. $B$ : preand postlearning orientation tuning curves of a cell whose preferred orientation $\left(49^{\circ}\right)$ is far from the learned orientation. Leaning causes modest broadening of the tuning curve on the side facing the learned orientation. $C$ : slopes of cells' tuning curves at the learned orientation $\left(0^{\circ}\right)$ before and after learning. Note that the greatest increase in slope occurs for cells whose prelearning slope is the highest at the learned orientation. $D$ : peak shift of orientation tuning curves caused by learning. A shift toward the trained orientation is defined as negative. In both $C$ and $D$, the $x$ axis is the cells' preferred orientations before learning. Simulation parameters were the same as for Fig. 3 . after learning (Fig. 4B). The point at which orientation tuning curves ceased to show a "near" pattern of sharpening and began to show a "far" pattern of broadening depended on the parameter set used in a given simulation, particularly the intracortical interaction profile. Cells with a preferred orientation very far away from (i.e., nearly orthogonal to) the trained orientation showed little changes to their tuning curves. As we will detail below, these features compare well with the physiological data of Schoups et al. (2001).

This specific pattern of tuning sharpening and broadening can be understood intuitively. Consider the three orientation tuned cells marked 1, 2, and 3 in Fig. 3, with cell 1 tuned to the trained orientation. The Mexican-hat interaction profile among the cells (Fig. 1C) means that cells 1 and 2 excite each other while cells 1 and 3 inhibit each other. Therefore part of the response of cell 2 at the trained orientation is due to the excitation from cell 1. Similarly, the diminished response of cell 3 at the trained orientation is partly due to the inhibition from cell 1 . Since orientation learning causes a long-lasting reduction of neuronal activities at and around the trained orientation, cell 2 will lose some excitation at the trained orientation and thus have a sharper tuning curve, while cell 3 will lose some inhibition and become more broadly tuned. Thus an activity reduction at and around the trained orientation in the recurrent model will lead to the specific pattern of tuning curve sharpening and broadening due to the disturbance of the balance of excitation and inhibition in the network. The sharpening and broadening in the orientation domain discussed here are analogous to the receptive field contraction and expansion in the spatial domain reported by Pettet and Gilbert (1992). During the learning process, cells with preferred orientations further away from the stimulus orientations are initially silent and are therefore in an "orientation scotoma," and their orien- tation tuning curves expand (broaden) over time. In contrast, those with preferred orientations near the stimulus orientations are repeatedly stimulated, and their orientation tuning curves contract (sharpen).

Schoups et al. (2001) observed trends for both the "near" sharpening and "far" broadening of orientation tuning curves predicted above in their physiological data. However, since the broadening effect failed to reach statistical significance, the data analysis was focused on the sharpening. To facilitate comparison between the model and the data, we measured the slope at the learned orientation for all the model cells, both before and after learning (in units of spikes $/ \mathrm{s} /{ }^{\circ}$ ). The result, shown in Fig. $4 C$, well replicates the physiological counterpart in Fig. $2 C$ of Schoups et al. (2001). In particular, the physiological data showed that cells with the steepest slope at the trained orientation before learning are the ones with the greatest increase in slope after learning. This is duplicated by our model, as the highest portion of the prelearning slope (dashed curve) in Fig. $4 C$ has the greatest increase after learning (solid curve). This fact is significant for the following reason. Tuning curves with the steepest slope at the learned orientation will show the greatest change in firing to small orientational changes around this orientation. Therefore these cells could be the most important for distinguishing small differences in orientation at the learned orientation (Albrecht and Geisler 1997; Lehky and Sejnowski 1990; Regan and Beverley 1985). If these are the same cells whose tuning curve slope at the learned orientation increases the most after learning, this could explain the improved psychophysical performance of subjects after learning. We will examine this issue more closely with signal detection theory below. With our standard parameter set, our model predicts that tuning curve sharpening occurs for cells with preferred orientations between $18^{\circ}$ and $30^{\circ}$ away 
from the learned orientation; Schoups et al. (2001) found this range to be between $12^{\circ}$ and $20^{\circ}$.

The far broadening predicted by the model can also contribute to the performance improvement at the trained orientation. The reason is that before learning, these far cells have practically no response near the trained orientation and therefore cannot contribute to the discrimination of two slightly different orientations around the trained orientation. After learning, the curve broadening allows the cells to reach over and have some responses near the trained orientation and thus contribute to the discrimination. However, since the responses from the far cells are small at the trained orientation, the contribution to the performance improvement around the trained orientation by the far cells through broadening is much less than that by near cells through sharpening.

Figure $4 C$ is symmetric with respect to the trained orientation. Thus the cell with the highest slope at the trained orientation can be found on either side of the trained orientation. This highest slope is 2 before learning. After learning, this value ranges from 2.5 to 5.2 for various combinations from our standard parameter set. Schoups et al. (2001) normalized their orientation tuning curves and found the highest slope to be about $2-2.5 \%$ change in firing per degree before learning and about $3 \%$ change in firing per degree after learning. Normalizing our values in the same fashion gave a prelearning maximum slope of 5\% change per degree, and a postlearning range of $5.5-13.5 \%$ change per degree. Thus, although our postlearning slope values were higher than the values from Schoups et al. (2001), our model's prelearning slope was higher as well, and the ratio of the pre- and postlearning slopes is similar in both cases.

We also quantified the peak shift of the cells in our model. The shift caused by learning as a function of the preferred orientation before learning is plotted in Fig. 4D. Here, we have defined shifting toward the trained orientation as a negative shift and shifting away from the trained orientation as positive shift. Negative shift was observed after learning in our model. For various parameter combinations in the standard set, the maximum peak shift tended to occur for cells whose preferred orientations before learning were between $20^{\circ}$ and $40^{\circ}$ away from the trained orientation, and the maximum shift magnitude varied from $4.2^{\circ}$ to $12.4^{\circ}$. There are no physiological data we can compare these simulation results with, because in the physiological experiments of Schoups et al. (2001), the learned and control cells were different populations of cells because it is impossible to hold the same cells for recording through months of training process. The peak shift is thus an untested prediction of the model.

\section{Adaptation}

The baseline simulation of the model before adaptation is identical to that before learning, and the result is duplicated in Fig. 5A. We simulated adaptation by depressing both intracortical excitatory and intracortical inhibitory connections around the trained orientation by a large fraction (see METHODS). The consequence of the manipulation was a reduction of net excitation at the peak and a reduction of net inhibition at the troughs of the Mexican-hat interaction profile for cells around the adapted orientation.

As in the learning simulations, adaptation depressed the amplitude of orientation tuning curves for cells whose preferred orientations were near the adapted orientation (Fig. 5B). The maximum reduction value for the simulation in Fig. 5 is $19.7 \%$ at the adapted orientation, and our standard parameter set for adaptation gave maximum reduction values ranging from $10.0 \%$ to $49.4 \%$. These values are in accordance with Dragoi et al. (2000), who reported max reduction values of over $40 \%$. In addition to depressing response amplitudes, simulating adaptation in the recurrent model broadened orientation tuning curves for cells with preferred orientations near the adapted orientation and shifted peaks away from the adapted orientation (Fig. 6A), all in accordance with the physiological data for adaptation by Dragoi et al. (2000). Another related change to the tuning curve in Fig. $6 A$ was that on the side of the curve facing away from the adapted orientation (termed the far side of the tuning curve hereafter), the responses were stronger after adaptation so that over this portion, the postadaptation curve was above the preadaptation curve. This is again in agreement with the physiological data (Dragoi et al. 2000). Finally, Dragoi et al. (2000) found statistically insignificant sharpening for tuning curves of cells whose preferred orientation was more than $60^{\circ}$ away from the adapted orientation. We looked at orientation tuning curves in this range in our adaptation simulations, and we also found modest sharpening for these curves (Fig. 6B).

For both learning and adaptation, there is a neural activity reduction around the trained or adapted orientation. This was reproduced in our simulations by a reduction of net excitation (the positive part of the Mexican-hat interaction profile in Fig. $1 C)$ to cells around the trained or adapted orientation. However, learning leads to near sharpening and (statistically insignificant) far broadening of tuning curves (Schoups et al. 2001)
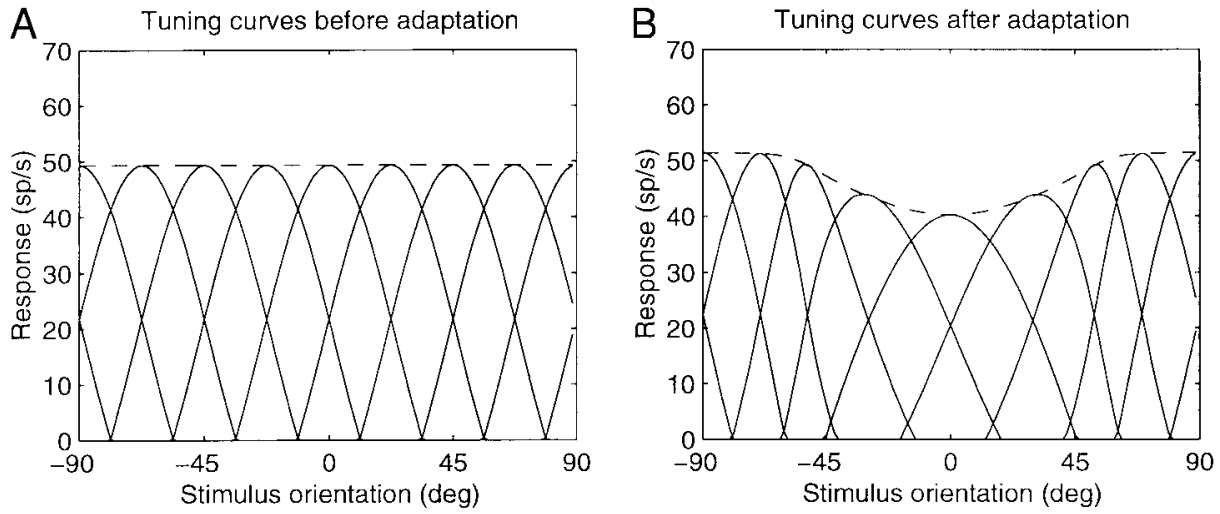

FIG. 5. Simulation of adaptation in the recurrent model. $A$ : orientation tuning curves for a subset of model V1 cells before adaptation. Dashed line was derived by plotting the peak response of every cell in the network. $B$ : orientation tuning curves for the same subset of cells after simulating adaptation at $0^{\circ}$. The peak response curve (dashed) shows a dip at the adapted orientation. This figure was obtained with an $A_{\mathrm{e}}$ of 0.2 , an $A_{\mathrm{i}}$ of 0.22 , and a $\sigma_{\mathrm{r}}$ of 20 . 

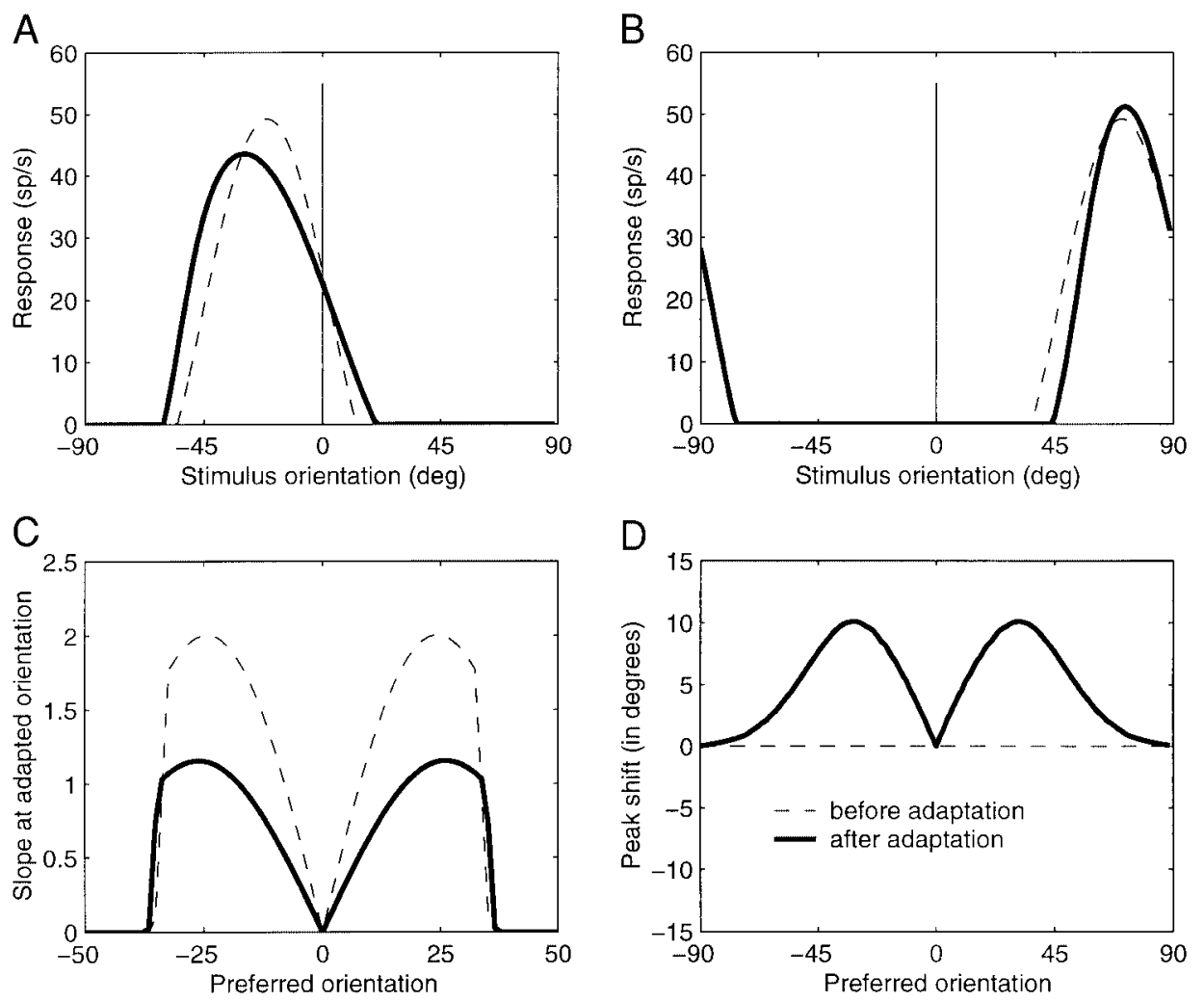

FIG. 6. Effects of adaptation in the recurrent model. This figure makes the effects of the adaptation simulation in Fig. 5 more explicit. Adaptation has been simulated at $0^{\circ}$ (marked by vertical lines). In all panels, dashed and solid lines represent the preadaptation and postadaptation conditions, respectively. $A$ : the preand postadaptation orientation tuning curves of a cell whose preferred orientation is close $\left(21^{\circ}\right)$ to the adapted orientation. Adaptation caused broadening of the tuning curve, with a smaller slope on the side facing the adapted orientation, and a shift of peak away from the adapted orientation. $B$ : pre- and postadaptation orientation tuning curves of a cell whose preferred orientation is far $\left(70^{\circ}\right)$ from the adapted orientation. Adaptation caused modest sharpening of the tuning curve on the side facing the adapted orientation. $C$ : slopes of cells' tuning curves at the adapted orientation $\left(0^{\circ}\right)$ before and after adaptation. $D$ : peak shift of orientation tuning curves caused by adaptation. A shift away from the adapted orientation is defined as positive. In both $C$ and $D$, the $x$ axis is the cells' preferred orientations before adaptation. Simulation parameters were same as for Fig. 5. while adaptation causes near broadening and (statistically insignificant) far sharpening (Dragoi et al. 2000). In addition, learning is predicted to shift tuning-curve peaks of near cells toward the trained orientation (Fig. 4), whereas adaptation shifts them away from the adapted orientation (Dragoi et al. 2000). We were able to reproduce these opposite features in our learning and adaptation simulations by introducing different modifications to the connections in the recurrent network. As we explained earlier, the reduction of net excitation alone can generate the features associated with learning. The main difference between our learning and adaptation simulations was that for the latter, the net inhibitory interaction (the negative part of the Mexican-hat in Fig. 1C) was reduced for cells around and somewhat away from the adapted orientation. This reduction of net inhibition was responsible for the enhanced responses on the far side of the tuning curves of the near cells (Fig. 6A). The enhanced responses, in turn, "pulled" the peak away from the adapted orientation and broadened the curve on the side facing the adapted orientation. In other words, the reductions of the net excitatory and the net inhibitory parts of the Mexican-hat interaction profile have opposite effects on the tuning curves. When the net-excitation reduction is dominant, learning related features will be observed. With progressively stronger reduction of the net inhibition, the learning-related features will be weakened, and the adaptation-related features will eventually emerge. Note that the reduction of net inhibition cannot be used alone to model adaptation; the reduction of net excitation is also necessary in the adaptation simulation to generate the dip of neural activity around the adapted orientation.

Every analysis we performed on the learning simulations was applied to the adaptation simulations. Thus we plotted in Fig. $6 C$ the slope of tuning curves at the adapted orientation against the cells' preferred orientation before adaptation. As expected, the broadening of the orientation tuning curves lowered the slope values. The highest slope at the adapted orientation before adaptation is 2; after adaptation, this value ranges from 1.7 to 0.8 for our standard parameter set. We also plotted peak shift caused by adaptation against the preferred orientation before adaptation (Fig. 6D). In keeping with the convention used in Fig. 4D, we have plotted peak shifting away from the adapted orientation as a positive shift. Positive peak shifting was reported in the adaptation study of Dragoi et al. (2000), and our Fig. $6 D$ closely resembles Fig. $1 E$ in their paper. Dragoi et al. (2000) reported a maximum shift of about $10^{\circ}$ for simple cells whose preferred orientation was between $5^{\circ}$ and $22.5^{\circ}$ away from the adapted orientation. Our standard parameter set gave maximum shift values that ranged from $1.6^{\circ}$ to $10^{\circ}$, and the range of cells where this maximum appears was from $25^{\circ}$ to $40^{\circ}$ away from the adapted orientation. In Fig. $1 E$ of their paper, Dragoi et al. (2000) show that the range from $22.5^{\circ}$ to $45^{\circ}$ also has a high shift value, which is nearly as high as the maximum in the $5^{\circ}$ to $22.5^{\circ}$ range, and the error bars for the shift values of these two groups of cells largely overlap.

\section{Orientation discriminability after learning and adaptation}

We have shown above that our simulations can reproduce the main features of the physiological experiments for both learning and adaptation. We now present our studies on whether and how these physiological changes may be related to the behavioral consequences of learning and adaptation observed psychophysically.

The main behavioral consequence of orientation learning is an improvement of orientation discrimination at the trained orientation. As we mentioned earlier, the tuning curve changes 
predicted by our model and confirmed by the physiological experiments should be able to explain the psychophysically observed performance improvement at the trained orientation. To make this statement more quantitative, we used signal detection theory (Green and Swets 1966) to relate cells' population responses to a performance measure for an orientation discrimination task (see METHODS). We considered the discrimination of two orientations differing by $1.5^{\circ}$ and presented for $200 \mathrm{~ms}$ around the trained orientation. In Fig. $7 A$, we plot the percent correct performance as a function of the maximum activity reduction at the trained orientation. The leftmost point corresponds to the performance before learning. The figure shows that the performance increases as the activity reduction increases.

We also examined how training at one orientation may affect performances at other orientations in the model by placing the $1.5^{\circ}$ orientation difference at a full $180^{\circ}$ range of orientations (Fig. $7 B$, bold curve). For comparison, we also show the corresponding curve before learning (thin curve), which is largely flat (we did not include the oblique effect in the baseline model as it is irrelevant to the current study). The figure indicates that training at one orientation should not affect performance at the orthogonal orientation, consistent with psychophysical observations (Schoups et al. 1995). The curves also suggest that there should be a positive transfer to orientations near the trained orientation, and a smaller amount of negative transfer to orientations further away.

Finally, we also applied the signal detection theory procedure to our adaptation simulations. The result (data not shown) was a decrease in performance at the adapted orientation below baseline and a moderate increase in performance for orientations adjacent to the adapted orientation; in other words, the precise opposite from the learning simulation and the adaptation psychophysical literature. Therefore according to signal detection theory, the psychophysical observation of Regan and Beverley (1985) cannot be explained by the physiological data of Dragoi et al. (2000) (see DISCussion).

Although we used signal detection theory above, qualitatively identical conclusions can be reached with other methods that rely on the differences between neurons' responses to the two orientations being discriminated. For example, one could

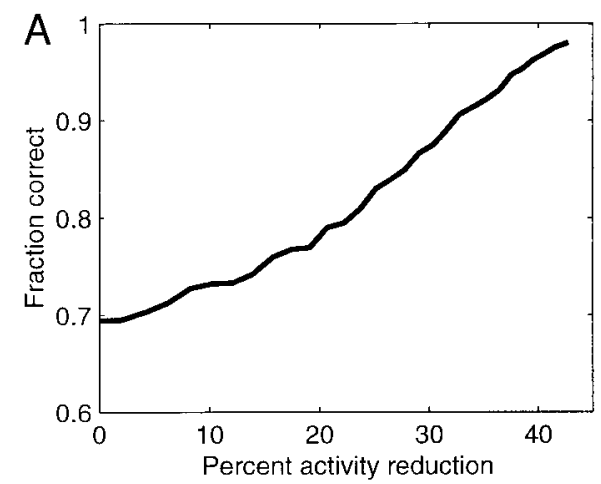

simply assume that the psychophysical performance is a monotonic function of the sum of the squared response differences (or the absolute values of the differences) from all cells. On the other hand, there are also methods that do not seem sensible. For instance, if one pools the response differences of all cells without first squaring them or taking absolute values, the differences of opposite signs will cancel each other, leading to almost no discriminability both before and after learning or adaptation.

In addition to signal detection theory, another well-known method is Bayesian analysis. Bayesian method predicts that the sharpening (broadening) of tuning curves will improve (impair) orientation discriminability because the sharpness of tuning determines the sharpness of the posterior probability distributions. Therefore the tuning-curve change for best improving performance under Bayesian method is near sharpening but not concurrent far broadening. Although powerful and useful for many applications, we believe that the Bayesian approach is problematic as a method for relating neuronal activities to psychophysical performances. The reason is that to estimate the posterior probability distributions, Bayesian method has to make the unreasonable assumption that at all times, the cells "know" their responses to all orientations including those that are not present in the current trial. In contrast, signal detection theory only requires the responses to the two stimulus orientations being compared.

\section{I S C U S S I O N}

The ultimate goals of this computational study are two-fold. First, we would like to understand the changes to V1 orientation tuning curves generated by learning and adaptation in the orientation domain. Second, we are interested in relating the physiologically observed changes to the perceptual consequences of orientation learning and adaptation. The results reported in this paper demonstrate that a physiologically based model can help bring us closer to these goals. At the same time, the effort also raises many new questions. In the following discussion, we summarize our main findings and discuss some related studies and the key open issues.

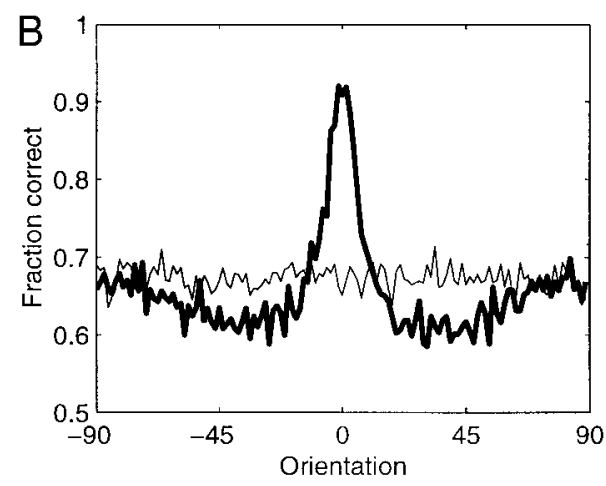

FIG. 7. Signal detection theory applied to the learning simulations. A: fraction of correct responses for discriminating an orientation difference of $1.5^{\circ}$ at the trained orientation, plotted as a function of the percent neural activity reduction at the trained orientation. The larger the activity reduction, the better the performance. The leftmost point corresponds to the performance before learning. Each point was estimated from 10,000 trials. The curve was obtained with a $\sigma_{\mathrm{r}}$ of 20, and $A_{\mathrm{e}}$ varied from 0 (leftmost point) to 0.02 (rightmost point). $B$ : transfer of learning at one orientation $\left(0^{\circ}\right)$ to other orientations. The $1.5^{\circ}$ orientation difference was placed at 128 evenly spaced orientations in the entire $180^{\circ}$ range. Each point was estimated from 1,000 trials. The simulation was run twice; once with postlearning neuronal responses (bold line) and once with prelearning neuronal responses (thin line). The learning simulation was run with an $A_{\mathrm{e}}$ of 0.015 and a $\sigma_{\mathrm{r}}$ of 20 . 


\section{Physiological comparisons}

Our modeling on changes of V1 orientation tuning curves has been focused on two physiological studies, the orientation learning study of Schoups et al. (2001) with behaving monkeys and the orientation adaptation study of Dragoi et al. (2000) with anesthetized cats. Our main finding is that the physiologically observed changes in V1 from both studies can be understood in the framework of the recurrent model of V1 orientation selectivity. In the case of learning, the reduction of neuronal firing rate and the sharpening of orientation tuning curves near the trained orientation can be accounted for by slightly reducing net recurrent cortical excitation to cells around the trained orientation. In the case of adaptation, the reduction of neuronal firing rate, the broadening of the tuning curve at the adapted orientation, and the peak shift away from the adapted orientation, can be reproduced by significantly reducing both recurrent excitation and recurrent inhibition to cells around the adapted orientation such that both the peak (net excitation) and the troughs (net inhibition) in the Mexican-hat interaction profiles had reduced amplitudes. The utility of our modeling effort is supported by the fact that we predicted the tuning curve changes caused by learning (Qian and Matthews 1999) before the physiological data were re-analyzed to confirm the prediction (Schoups et al. 2001).

Very recently, Ghose et al. (2002) reported that the only change they could find in monkey V1 after orientation learning was a small firing rate reduction around the trained orientation and that they failed to find the slope change in orientation tuning curves reported by Schoups et al. (2001). The difference between the two physiological studies may be explained by the difference in the learning paradigms employed. In the experiment of Schoups et al. (2001), the monkeys were trained to discriminate a grating orientation from the $45^{\circ}$ diagonal in each trial. This paradigm had been applied to human subjects previously, and like humans, the monkeys showed the orientation and location specificities typically associated with orientation perceptual learning. Ghose et al. (2002) also trained monkeys to discriminate orientations of grating stimuli presented at a retinal eccentricity similar to the Schoups et al. (2001) study $\left(3^{\circ}\right.$ vs. $\left.3.2^{\circ}\right)$. However, in addition to grating orientation, the spatial frequency of the gratings was also varied from trial to trial. Therefore the monkeys in their experiment had to learn to discount the frequency variation, a task different from orientation discrimination. Thus there is a possibility that the learning displayed by their monkeys may partially be attributed to learning to discount the frequency variation. This possibility is supported by two pieces of evidence. First, the monkeys in their experiment did not show the typical location specificity after perceptual learning. Indeed, learning to ignore the frequency variations may be more conceptual than perceptual in the sense that it is more related to task understanding than to pushing perceptual threshold of orientation discrimination. Second, their monkeys' postlearning threshold, defined at $79 \%$ correct performance, was $4^{\circ}-5^{\circ}$, whereas the threshold for Schoups et al.'s monkeys, defined at $85 \%$ correct performance, was about $0.6^{\circ}-1.2^{\circ}$. This is another indication that perhaps their monkeys did not learn as much about orientation discrimination per se as the monkeys of Schoups et al. (2001). Therefore their failure to find slope changes in V1 orientation tuning curves might be due to the lack of sufficient perceptual learning in orientation discrimination; conceptual learning of discounting frequency variation would not be expected to affect orientation tuning.

In the case of adaptation, there is also a physiological report that appears to be different in at least one aspect from the study of Dragoi et al. (2000) modeled in this paper. Recording from V1 of anesthetized monkeys, Müller et al. (1999) found that for cells preferring orientations near the adapted orientation, the adaptation process shifted the peak locations of the tuning curves away from the adapted orientation, in agreement with Dragoi et al. (2000). However, contrary to the broadening reported by Dragoi et al. (2000), Müller et al. (1999) found sharpening of tuning at the adapted orientation. We have been unable to find a connection modification scheme in the recurrent model that could shift the peak away from the adapted orientation and sharpen the slope of tuning curves at the adapted orientation at the same time. One difference between the two physiological studies on adaptation is species: Dragoi et al. (2000) used cats while Müller et al. (1999) used monkeys. A related difference is that monkey V1 appears to have significantly fewer simple cells than cat V1. In this aspect, it is interesting to note that Müller et al. (1999) reported that the tuning curve changes they found only occurred to complex cells, and the 10 simple cells they encountered showed no changes at all. Dragoi et al. (2000), on the other hand, recorded from 130 cells; 88 of them showed tuning curve changes, and some of these must be simple cells. Finally, Dragoi et al. (2000) adapted their cells for as long as $10 \mathrm{~min}$, while Müller et al. (1999) only adapted their cells for $\leq 0.5$ s. Further experimental investigations are needed for pinpointing the main factors responsible for the contradictions between these two physiological studies.

\section{Psychophysical comparisons}

We have shown that according to signal detection theory, the learning-induced changes in orientation tuning curves, predicted by our model (Qian and Matthews 1999) and confirmed by Schoups et al. (2001), are precisely what is needed for explaining the improved discriminability at the trained orientation. Specifically, after learning, the population responses to two slightly different orientations around the trained orientation can better differentiate the two orientations. Based on our simulation results, we hypothesize that during the training process, there is an increasingly larger firing rate reduction at and around the trained orientation. This leads to progressively stronger changes to orientation tuning curves, which in turn results in better orientation discrimination at the trained orientation, as shown in Fig. 7A. Although the tuning-curve sharpening of near cells and the broadening of far cells can both help improve orientation discriminability at the trained orientation, we find that the contribution of the near sharpening is more important than that of the far broadening. This is due to the fact that the broadening is not as pronounced as the sharpening because inhibition is not as strong as excitation in the Mexicanhat interaction profile among the V1 cells tuned to different orientations. In addition, the firing rate of the far cells at the trained orientation is much lower than that of the near cells. These considerations may also help explain that the broadening of far cells in the data of Schoups et al. (2001) failed to reach statistical significance. 
Our model also predicts that perceptual learning at one orientation should not affect performance at the orthogonal orientation (see Fig. 7B), consistent with psychophysical experiments on orientation learning (Schoups et al. 1995). In addition, the model predicts a positive transfer of learning to similar stimulus orientations and a smaller, negative transfer to orientations somewhat further away. The transition point from positive to negative transfer depends on the specific pattern of tuning curve sharpening and broadening, which in turn depends on the details of the Mexican-hat interaction profile. The negative transfer may be difficult to detect because it is smaller than the positive transfer and because there is often a small, nonspecific component in perceptual learning experiments (caused by the improved task familiarity through the training process) that may mask the small negative transfer predicted in Fig. $7 B$.

As mentioned in the Introduction, it is also known that immediately after orientation adaptation, the orientation discrimination at the adapted orientation is improved in human subjects (Regan and Beverley 1985). This psychophysical observation cannot be explained by the physiological findings of Dragoi et al. (2000), who found a broadening of tuning curves at the adapted orientation in anesthetized cats. We have confirmed this obvious conclusion by applying signal detection theory to our adaptation simulations. The adaptation data of Müller et al. (1999) from anesthetized monkeys may explain the improved discriminability at the adapted orientation, but as we will discuss next, their data fail to explain the tilt aftereffect, another perceptual consequence of adaptation. These discrepancies may not be too surprising given the enormous difference between the psychophysical adaptation studies with attending human subjects performing a difficult discrimination task and the physiological adaptation studies on anesthetized animals. Interestingly, the psychophysical effects of adaptation on discrimination reported by Regan and Beverley (1985) can be very well explained by our learning simulations shown in Fig. $7 B$. In particular, the positive and negative transfer profile in Fig. $7 B$ resembles Fig. 3 in their paper. Furthermore, our Fig. $7 B$ can explain that adaptation at one orientation does not affect discrimination at the orthogonal orientation (Westheimer and Gee 2002). (A previously reported effect of orthogonal adaptation (Clifford et al. 2001) has been shown to result from improper control of training (Westheimer and Gee 2002). A related effect using a very different paradigm, where the "adapting" stimulus temporally separates the two test stimuli being compared (Dragoi et al. 2002), is more likely due to orientation-specific masking than to adaptation.)

These considerations suggest that human adaptation results can be better accounted for by the physiological data of learning from behaving monkeys (Schoups et al. 2001) than by the physiological data of adaptation from anesthetized animals (Dragoi et al. 2000). The implication is that orientation adaptation in alert subjects may be viewed as a short-term version of orientation learning. It may be easier to observe the negative transfer predicted in Fig. $7 B$ in an adaptation experiment (Regan and Beverley 1985) than in a learning experiment because there is no lengthy training process involved in adaptation studies to generate any nonspecific improvement. A prediction is that, in behaving monkeys, the effect of adaptation should be more similar to the learning experiments of Schoups et al. (2001). [A very recent study measured adapta- tion effects with alert monkeys (Dragoi et al. 2002), but the adapting and test orientations were unrelated to animals' behavior. The effects are similar to those in Dragoi et al. (2000) and can thus be explained by our adaptation simulations.]

Another effect of orientation adaptation is the tilt aftereffect: adapting to one orientation makes subsequently presented nearby orientations appear to be rotated away from the adapted orientation (Gibson 1933; Wolfe 1984). Intuitively, one might think that the observed shift of the tuning-curve peaks away from the adapted orientation in anesthetized animals (Dragoi et al. 2000; Müller et al. 1999) could explain the tilt aftereffect. However, it has been pointed out previously that precisely the opposite is likely to be true (Gilbert and Wiesel 1990; Yao and Dan 2001). The key point is that it is the peak location (or weighted average orientation) of the population response, instead of the tuning curve, that determines the perceived orientation. The tilt aftereffect requires that the population responses (to stimulations near the adapted orientation) be shifted away from the adapted orientation. This in turn requires that the tuning curves around the adapted orientation be shifted toward the adapted orientation (Gilbert and Wiesel 1990; Yao and Dan 2001). It has also been noted that tuning-curve sharpening of the near cells can help (Gilbert and Wiesel 1990). These requirements are precisely satisfied by our learning simulations. However, our adaptation simulations and the adaptation data from anesthetized animals (Dragoi et al. 2000; Müller et al. 1999) all show a shifting-away of the tuning curves and therefore cannot explain the tilt aftereffect.

To make the above considerations more specific, we plotted the postlearning (Fig. 8, top panels) and postadaptation (Fig. 8, bottom panels) population responses of our model to a stimulus orientation $14^{\circ}$ away from the trained or adapted orientation. (The corresponding prelearning/adaptation population responses peak precisely and symmetrically at the stimulus orientation and are thus not shown.) Each population response is plotted in two ways: the activity of a given cell can be plotted at either 1 ) its preferred orientation before learning/adaptation (Fig. 8, $A$ and $C$ ), or 2) its preferred orientation after learning/ adaptation (Fig. 8, $B$ and $D$ ). If the perceived orientation corresponds to the peak location, then only the learning plot in Fig. $8 \mathrm{~A}$ can explain the tilt aftereffect. If the perception corresponds to the weighted average orientation (arrows) instead, then the learning plots in either Fig. 8, $A$ or $B$, can explain the illusion. The adaptation plots, on the other hand, either show little effect or an effect in the wrong direction. These results reinforce our above suggestion that adaptation in alert subjects may be better viewed as a short-term version of learning and cannot be explained by the adaptation data or simulations for anesthetized animals. We predict that adaptation in alert subjects performing an orientation discrimination task must generate a short-term shift of population activity like those shown in Fig. $8 A$ (or equivalently, a short-term change of tuning curves shown in Fig. 3) from our learning simulations.

There is a large asymmetry in the population response of Fig. $8 B$, with the right side of the curve dropping faster than the left side. This is because the postlearning preferred orientations are shifted toward the trained orientation $\left(0^{\circ}\right)$ and the shifts are larger for cells near the trained orientation (Fig. 4D). Likewise, the slower drop on the right side in Fig. $8 D$ is due to the shift-away of the postadaptation preferred orientations from the adapted orientation (Fig. 6D). The peak shift also explains why 

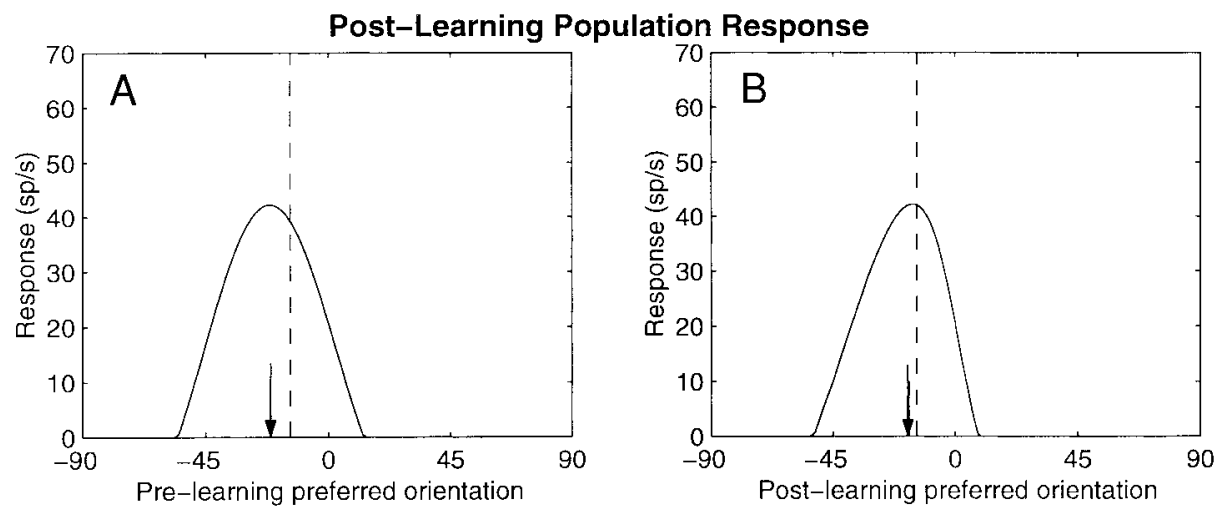

FIG. 8. Population responses after learning and adaptation. All 4 graphs show postlearning/ adaptation population responses of all model cells to a stimulus orientation of $-14^{\circ}$ (marked by dashed vertical lines). The learned/adapted orientation was $0^{\circ}$. $A$ and $B$ : population responses after learning. $C$ and $D$ : population responses after adaptation. In $A$ and $C$, the preferred orientation of each cell before learning/ adaptation was used as the $x$ axis. In $B$ and $D$, the preferred orientation of each cell after learning/ adaptation was used as the $x$ axis. Arrows indicate the weighted average orientation of the distributions. Learning was simulated with an $A_{\mathrm{e}}$ of 0.075 and a $\sigma_{\mathrm{r}}$ of 24 . Adaptation was simulated with an $A_{\mathrm{e}}$ of 0.2 , an $A_{\mathrm{i}}$ of 0.22 , and a $\sigma_{\mathrm{r}}$ of 20 .
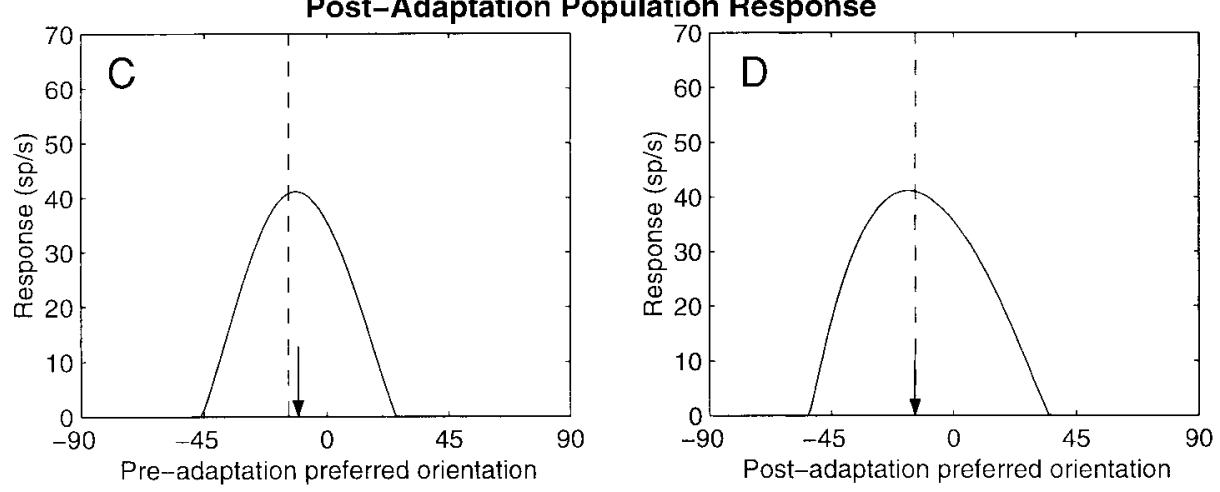

the curves in Fig. 8, $A$ and $C$, are wider and narrower than those in Fig. 8, $B$ and $D$, respectively.

Which way of plotting population responses in Fig. 8 is more reasonable? Suppose a cell prefers orientation $\theta_{\text {pre }}$ before adaptation, and for a short period after adaptation, its preferred orientation shifts to $\theta_{\text {post }}$. The critical question is this: when this cell fires after adaptation, does it signal the presence of $\theta_{\text {pre }}$ or $\theta_{\text {post }}$ in the stimulus? While there is no known answer, we believe that in the case of adaptation, the cell must always signal $\theta_{\text {pre }}$ rather than $\theta_{\text {post }}$. The reason is that the meaning of a cell's activity is most likely determined by its projections to higher centers. This connectivity pattern is largely shaped when the cell's preferred orientation is $\theta_{\text {pre }}$, and is unlikely to be altered by only a brief shift of the preference to $\theta_{\text {post }}$ after adaptation. Thus, as far as the tilt aftereffect after adaptation is concerned, we should only consider panels Fig. 8, $A$ and $C$. The situation is less clear for perceptual learning because learninginduced changes are long-lasting and could lead to some reorganization downstream.

A better known consequence of orientation adaptation is the elevation of contrast detection threshold (Regan and Beverley 1985). This can be explained by the peak firing rate reduction at the adapted orientation. It is not ad hoc to use the slope of the orientation tuning curves to explain orientation discriminability, and the peak firing rate to account for contrast detection threshold. In both cases, we assume that the brain relies on signals that can best perform the respective tasks. Specifically, in the discrimination task, two similar orientations with high luminance contrast (well above contrast threshold) are compared, while in the detection task, the presence or absence of a stimulus with low luminance contrast (near contrast threshold) is judged. In either task, the best performance is achieved by cells with the largest differential responses to the conditions being compared. For the discrimination task, cells with the steepest tuning slopes at the stimulus orientations give the largest differential responses to the two stimuli. For the detection task, on the other hand, cells with maximum responses at the stimulus orientation give the largest differential responses to the presence and absence of the stimulus. Therefore, while orientation-discrimination learning should lead to a tuningcurve sharpening near the trained orientation (Schoups et al. 2001), contrast-detection learning should lead to an increased peak firing rate. A recent fMRI study is consistent with this prediction (Furmanski and Engel 2002).

\section{Synaptic mechanisms}

In this paper, we have focused on how to model the end effects of learning and adaptation observed physiologically by altering connections in a recurrent network of V1 orientation selectivity. We have not studied what kind of synaptic plasticity rules could produce the required connection changes. While this is an interesting question that deserves investigation, here we only briefly argue that the general features of the connection changes (Eqs. 8 and 9) proposed in our model are not unreasonable. First, our model mainly requires synaptic depression of the recurrent connections. Mechanisms for shortterm depression after repeated stimulation have been described (Abbott et al. 1997; Stratford et al. 1996; Tsodyks et al. 1998; Varela et al. 1997) and may be responsible for the transient decrease of connection strengths needed in our adaptation simulations. Long-term depression (LTD) of synaptic connections needed in our learning simulations has also been documented (Artola and Singer 1990; Bear et al. 1987; Fregnac et al. 1994; Kirkwood et al. 1996); these studies indicate that synaptic modifications can switch from long-term potentiation 
(LTP) to LTD as the postsynaptic activity decreases below a certain sliding threshold. Second, our model requires that for both recurrent excitatory and inhibitory connections (the solid and dashed curves in Fig. 1B), the largest reduction is for cells tuned to the trained or adapted orientation and the reduction gradually tapers off for cells whose preferred orientations are further away (Eqs. 8 and 9). This makes sense because cells tuned to the trained or adapted orientation fire most during the learning or the adaptation process and should thus experience the strongest modification. Note that in our adaptation simulation, the net reduction of inhibition in the troughs of the Mexican-hat is not achieved by a larger reduction of inhibition for cells away from the adapted orientation than for cells tuned to the adapted orientation; the opposite is true. Rather, the net inhibition reduction occurs at the troughs instead of at the peak of the Mexican hat, because at the peak, the concurrent excitation reduction more than compensates for the inhibition reduction and the excitatory connection profile is narrower than the inhibitory one. Third, we would like to mention that some aspects of Schoups et al.'s (2001) learning data and Dragoi et al.'s (2000) adaptation data can also be reproduced by changing the feed-forward connections of the model in an orientation-dependent manner. However, we did not report any of these simulations because the presynaptic cells of the feedforward connections are not orientation tuned and are therefore unlikely to support an orientation-dependent synaptic modification mechanism. Finally, we predicted that the adaptationinduced changes in alert subjects performing a discrimination task and in anesthetized animals must be quite different. This is not far fetched as there is evidence suggesting that synaptic plasticity can be dependent on behavioral context (Ahissar et al. 1992) and that the physiological substrate of learning in alert monkeys can be task dependent (Crist et al. 2001). We speculate that the connection changes used in our adaptation simulations never happen to behaving subjects performing an orientation discrimination task; instead, short-term adaptive changes similar to our learning simulations first occur, and these changes gradually become permanent during the long training process.

\section{Models of V1 orientation selectivity}

In addition to the interesting problems of learning and adaptation, our work is also relevant to the on-going debate on the mechanisms of orientation tuning in V1 (Ferster and Miller 2000; Sompolinsky and Shapley 1997). In their classical studies of orientation selectivity, Hubel and Wiesel (1962) proposed a feed-forward model, which posits that oriented V1 cells receive inputs from several LGN cells with properly aligned, center-surround receptive fields (Reid and Alonso 1995). However, to accommodate a large number of related physiological observations, such as the contrast invariance of orientation tuning and the effects of inhibition blocking (see Somers et al. (1995) for a thorough discussion), several groups proposed the recurrent model adopted in this paper, which assumes a weak orientation bias via the feed-forward mechanism of Hubel and Wiesel (1962) and a subsequent sharpening of the tuning via intracortical excitation and inhibition (BenYishai et al. 1995; Carandini and Ringach 1997; Douglas et al. 1995; Somers et al. 1995). As we explained in RESULTS, the learning- and adaptation-induced changes simulated in this study rely on the recurrent interactions (see also Dragoi et al. (2000)). A strictly feed-forward model cannot predict those changes since modification of the feed-forward connections would only change the overall amplitude of V1 responses without generating the specific pattern of broadening and sharpening or peak shifts of the tuning curves observed physiologically.

Our work also helps alleviate two criticisms against the recurrent model: we showed that the model still works when the recurrent excitatory and inhibitory profiles are made similar (but not identical), and that when the parameters are more consistent with physiology, the model does not generate spurious peaks under the noise condition (see METHODS). However, other problems with the recurrent model, such as the inconsistency with the cortical inactivation experiments and the near independence of tuning on stimulus' spatial frequency, have been noted (Ferster and Miller 2000) and have prompted Troyer et al. (1998) to propose a modified feed-forward model. According to this model, V1 orientation tuning mainly results from the feed-forward mechanism and the contrast invariance is maintained by feed-forward inhibition between cells with opposite receptive field polarities. While successful in many ways (Kayser et al. 2001; Krukowski and Miller 2001; Troyer et al. 1998), the modified model is unlikely to explain the learning- and adaptation-induced changes because it does not assume a Mexican-hat interaction profile among cells tuned to different orientations. It is also not clear if the modified feedforward model is consistent with some other phenomena accounted for by the recurrent model (Somers et al. 1995; Sompolinsky and Shapley 1997).

Perhaps the truth lies somewhere between the recurrent model and the feed-forward model (Miller et al. 2001): the relative weighting between the feed-forward and recurrent contributions might be different for different V1 cells. We have made additional simulations by varying this weighting in the recurrent model and found that most results reported in this paper remain qualitatively the same under many different combinations of feed-forward and recurrent contributions to V1 tuning. The main exception is that the sharpening effect in the learning simulation does require a weak feed-forward tuning and a strong recurrent connection. It is interesting to note in this context that physiologically, the learning-induced sharpening was mainly found in superficial and deep layers (Schoups et al. 2001), where the recurrent connections may be more dominant than the input layer 4 , and that the cortical inactivation experiments were limited to simple cells (Ferster and Miller 2000), which may receive less recurrent connections than complex cells (Chance et al. 1999).

In conclusion, we have proposed a model for understanding the V1 orientation tuning-curve changes induced by perceptual learning and adaptation, as reported by Schoups et al. (2001) and Dragoi et al. (2000), respectively. The two physiological studies found reduced neural activities around the trained or adapted orientation, but opposite patterns of changes to orientation tuning curves. We were able to account for the key features of both studies by introducing different modifications to the connections in a recurrent network for V1 orientation selectivity (Carandini and Ringach 1997; Somers et al. 1995). We also applied signal detection theory to quantify the perceptual consequences of the tuning curve changes and compared the results with the relevant psychophysical data. The learning- 
induced tuning curve changes can not only explain the psychophysical consequences of learning, but also the psychophysical consequences of adaptation, if the same changes are assumed to be long-lasting for learning but short-term for adaptation. In contrast, the adaptation data of Dragoi et al. (2000) cannot explain the altered orientation discriminability (Regan and Beverley 1985) or the tilt aftereffect observed after adaptation. A related physiological study on adaptation by Müller et al. (1999) also fails to explain the tilt aftereffect. We hypothesize that the discrepancies in the case of adaptation are caused by the difference between the psychophysical experiments with attending subjects and the physiological studies with anesthetized animals. We predict that with behaving animals performing an orientation discrimination task, the adaptation-induced physiological changes should be more like a short-term version of the learning experiments of Schoups et al. (2001). Finally, we would like to point out that our approach is quite different from previous perceptual learning models (Herzog and Fahle 1998; Peres and Hochstein 1994; Poggio et al. 1992) that rely on training artificial neural networks through connectionist learning algorithms. Although interesting in their own right, those models have relatively limited implications for biological systems. The explanatory and predictive power of our model derives from its close relation to physiology.

We thank Drs. Y. Chen, A. Das, V. Ferrera, A. Gee, N. Matthews, G. Orban, A. Schoups, R. Vogels, and members of the Mahoney Center at Columbia for helpful discussions and comments. We are grateful to Drs. M. Carandini and D. Ringach for making the source code of their recurrent model available.

This work was supported by National Science Foundation Grant IBN 9817979 to N. Qian, and by National Institutes of Health Grants HD-07430 and $\mathrm{MH}-54125$.

\section{REFERENCES}

Abbott LF, Varela JA, Sen K, and Nelson SB. Synaptic depression and cortical gain control. Science 275: 220-224, 1997.

Ahissar E, Vaadia E, Ahissar M, Bergman H, Arieli A, and Abeles M. Dependence of cortical plasticity on correlated activity of single neurons and on behavioral context. Science 257: 1412-1415, 1992.

Albrecht DG and Geisler WS. Visual cortex neurons in monkeys and cats: detection, discrimination, and identification. Visual Neurosci. 14: 897-919, 1997.

Artola A and Singer W. Different voltage-dependent thresholds for inducing long-term depression and long-term potentiation in slices of rat visual cortex. Nature 347: 69-72, 1990.

Bear MF, Cooper LN, and Ebner FF. A physiological basis for a theory of synaptic modification. Science 237: 42-48, 1987.

Ben-Yishai R, Bar-Or RL, and Sompolinsky H. Theory of orientation tuning in the visual cortex. Proc Nat Acad Sci USA 92: 3844-3848, 1995.

Carandini M and Ringach DL. Prediction of a recurrent model of orientation selectivity. Vision Res. 37: 3061-3071, 1997.

Chance FS, Nelson SB, and Abbott LF. Complex cells as cortically amplified simple cells. Nature Neurosci 2: 277-282, 1999.

Clifford CWG, Wyatt AM, Arnold DH, Smith ST, and Wenderoth P. Orthogonal adaptation improves orientation discrimination. Vision Res. 41: 151-159, 2001.

Crist RE, Li W, and Gilbert CD. Learning to see: experience and attention in primary visual cortex. Nature Neurosci 4: 519-525, 2001.

Douglas RJ, Koch C, Mahowald M, Martin KA, and Suarez HH. Recurrent excitation in neocortical circuits. Science 269: 981-985, 1995.

Dragoi V, Sharma J, Miller EK, and Sur M. Dynamics of neuronal sensitivity in visual cortex and local feature discrimination. Nature Neurosci 5: 883-891, 2002.

Dragoi V, Sharma J, and Sur M. Adaptation-induced plasticity of orientation tuning in adult visual cortex. Neuron 28: 287-298, 2000.

Ferster D. Orientation selectivity of synaptic potentials in neurons of cat primary visual cortex. J Neurosci 6: 1284-1301, 1986.
Ferster D and Miller KD. Neural mechanisms of orientation selectivity. Annu Rev Neurosci 23: 441-471, 2000.

Fregnac Y, Smith JP, and Friedlander MJ. Temporal covariance of pre- and postsynaptic activity regulates function connectivity in the visual cortex. J Neurophysiol 71: 1403-1421, 1994.

Furmanski CS and Engel SA. Perceptual learning leads to increases in V1 activity. Soc Neurosci Abst (CDROM), 2002.

Ghose GM and Maunsell JHR. Perceptual learning can selectively alter neural responses primate V1. Soc Neurosci Abst 23: 1544, 1997.

Ghose GM, Yang T, and Maunsell JHR. Physiological correlates of perceptual learning in monkey V1 and V2. J Neurophysiol 87: 1867-1888, 2002.

Gibson JJ. Adaptation, after-effect and contrast in the perception of curved lines. J Exp Psychol 16: 1-33, 1933.

Gilbert CD. Early perceptual learning. Proc Nat Acad Sci USA 91: 11951197, 1994.

Gilbert CD and Wiesel TN. The influence of contextual stimuli on the orientation selectivity of cells in primary visual cortex of the cat. Vision Res 30: 1689-1701, 1990.

Green DM and Swets JA. Signal Detection Theory and Psychophysics. New York: John Wiley, Inc., 1996.

Herzog MH and Fahle M. Modeling perceptual learning: difficulties and how they can be overcome. Biol Cybern 78: 107-117, 1998.

Hubel DH and Wiesel T. Receptive fields, binocular interaction, and functional architecture in the cat's visual cortex. J Physiol 160: 106-154, 1962.

Kayser AS, Priebe NJ, and Miller KD. Contrast-dependent nonlinearities arise locally in a model of contrast-invariant orientation tuning. J Neurophysiol 85: 2130-2149, 2001.

Kirkwood A, Rioult MC, and Bear MF. Experience-dependent modification of synaptic plasticity in visual cortex. Nature 381: 526-528, 1996.

Krukowski AE and Miller KD. Thalamocortical NMDA conductances and intracortical inhibition can explain cortical temporal tuning. Nature Neurosci 4: 424-430, 2001.

Lehky SR and Sejnowski TJ. Neural model of stereoacuity and depth interpolation based on a distributed representation of stereo disparity. $\mathrm{J} \mathrm{Neu}$ rosci 10: 2281-2299, 1990.

Michalski A, Gerstein GI, Czarkowska J, and Tarnecki R. Interactions between cat striate cortex neurons. Exp Brain Res 51: 97-107, 1983.

Miller KD, Pinto DJ, and Simons DJ. Processing in layer 4 of the neocortical circuit: new insights from visual and somotosensory cortex. Curr Opin Neurobiol 11: 488-497, 2001.

Müller JR, Metha AB, Krauskopf J, and Lennie P. Rapid adaptation in visual cortex to the structure of images. Science 285: 1405-1408, 1999.

Peres R and Hochstein S. Modeling perceptual learning with multiple interacting elements: a neural network model describing early visual perceptual learning. J Comput Neurosci 1: 323-338, 1994.

Pettet MW and Gilbert CD. Dynamic changes in receptive field size in cat primary visual cortex. Proc Nat Acad Sci USA 89: 8366-8370, 1992.

Poggio T, Fahle M, and Edelman S. Fast perceptual learning in visual hyperacuity. Science 256: 1018-1021, 1992.

Qian N and Matthews N. A physiological theory for visual perceptual learning of orientation discrimination. Soc Neurosci Abst 25: 1316, 1999.

Regan D and Beverley KI. Postadaptation orientation discrimination. J Opt Soc Am A 2: 147-155, 1985.

Reid RC and Alonso JM. Specificity of monosynaptic connections from thalamus to visual cortex. Nature 378: 281-284, 1995.

Schoups AA, Vogels R, and Orban GA. Human perceptual learning in identifying the oblique orientation: retinotopy, orientation specificity and monocularity. J Physiol 483: 797-810, 1995.

Schoups AA, Vogels R, and Orban GA. Effects of perceptual learning in orientation discrimination on orientation coding in v1. Invest Ophthalmol Vis Sci Suppl 39: 684, 1998.

Schoups AA, Vogels R, Qian N, and Orban GA. Practising orientation identification improves orientation coding in V1 neurons. Nature 412: 549-553, 2001.

Shadlen MN and Newsome WT. Noise, neural code and cortical organization. Curr Opin Neurobiol 4: 569-579, 1994.

Shiu LP and Pashler H. Improvement in line orientation discrimination is retinally local but dependent on cognitive set. Percept Psychophysiol 52: 582-588, 1992.

Snowden RJ, Treue S, and Andersen RA. The response of neurons in areas V1 and MT of the alert rhesus monkey to moving random dot patterns. Exp Brain Res 88: 389-400, 1992. 
Softky WR and Koch C. The highly irregular firing of cortical cells is inconsistent with temporal integration of random EPSPs. J Neurosci 13: 334-350, 1993.

Somers DC, Nelson SB, and Sur M. An emergent model of orientation selectivity in cat visual cortical simple cells. J Neurosci 15: 5448-5465, 1995.

Sompolinsky H and Shapley R. New perspectives on the mechanisms for orientation selectivity. Curr Opin Neurobiol 7: 514-522, 1997.

Stratford KJ, Tarczy-Hornoch K, Martin KAC, Bannister NJ, and Jack JJB. Excitatory synaptic inputs to spiny stellate cells in cat visual cortex. Nature 382: 258-261, 1996.

Troyer TW, Krukowski A, Priebe NJ, and Miller KD. Contrast-invariant orientation tuning in cat visual cortex: feed-forward tuning and correlationbased intracortical connectivity. J Neurosci 18: 5908-5927, 1998.
Tsodyks M, Pawelzik K, and Markram H. Neural networks with dynamic synapses. Neural Comput 10: 821-835, 1998.

Varela JA, Sen K, Gibson J, Fost J, Abbott LF, and Nelson SB. A quantitative description of short-term plasticity at excitatory synapses in layer 2/3 of rat primary visual cortex. J Neurosci 17: 7926-7940, 1997.

Vogels R and Orban GA. The effect of practice on the oblique effect in line orientation judgments. Vision Res 25: 1679-1687, 1985.

Westheimer G and Gee A. Orthogonal adaptation and orientation discrimination. Vision Res 42: 2339-2343, 2002.

Wolfe JM. Short test flashes produce large tilt aftereffects. Vision Res 24: 1959-1964, 1984.

Yao H and Dan Y. Stimulus timing-dependent plasticity in cortical processing of orientation. Neuron 32: 315-323, 2001. 